\title{
On the Equivalence of Contraction and Koopman Approaches for Nonlinear Stability and Control
}

\author{
Bowen $\mathrm{Yi} \odot$, and lan R. Manchester
}

\begin{abstract}
In this paper we prove new connections between two frameworks for analysis and control of nonlinear systems: the Koopman operator framework and contraction analysis. Each method, in different ways, provides exact and global analyses of nonlinear systems by way of linear systems theory. The main results of this paper show equivalence between contraction and Koopman approaches for a wide class of stability analysis and control design problems. In particular: stability or stablizability in the Koopman framework implies the existence of a contraction metric (resp. control contraction metric) for the nonlinear system. Further in certain cases the converse holds: contraction implies the existence of a set of observables with which stability can be verified via the Koopman framework. We provide results for the cases of autonomous and timevarying systems, as well as orbital stability of limit cycles. Furthermore, the converse claims are based on a novel relation between the Koopman method and construction of a Kazantzis-Kravaris-Luenberger observer. We also provide a byproduct of the main results, that is, a new method to learn contraction metrics from trajectory data via linear system identification.
\end{abstract}

Index Terms-nonlinear system, contraction analysis, Koopman operator

\section{INTRODUCTION}

Learning, analysis, and control of nonlinear dynamical systems are important problems but are significantly more challenging than their linear counterparts. In recent years two approaches - based on the Koopman operator and contraction analysis - have become popular and facilitated significant progress. Each, in different ways, draws on linear systems theory to analyze nonlinear systems exactly and globally: the Koopman approach works by mapping the system state to high (possibly infinite) dimensional spaces of observables in which the dynamics are linear, whereas the contraction framework analyzes the system via an infinite family of local linearizations. The main purpose of this paper is to make precise the connection between these two approaches.

The Koopman operator describes the dynamics over the space of observables. Even for nonlinear dynamics, the Koopman operator itself is always linear, and this fact can be

This work was supported by Australian Research Council. (Corresponding author: Bowen Yi)

The authors are with Australian Centre for Field Robotics and Sydney Institute for Robotics and Intelligent Systems, The University of Sydney, Sydney, NSW 2006, Australia. (email: \{bowen.yi, ian.manchester\}@sydney.edu.au) utilized for many tasks including, e.g., stability analysis, controller design, and system identification; see [37] for a recent review.

Unlike linearization via first-order approximation, the Koopman method provides a global insight to the system behaviour via spectral analysis of the operator, which is built upon the pioneering work [17]. Furthermore, for a given system if we are able to find a finite set of Koopman eigenvalues and eigenfunctions, then the system can be immersed into a finite-dimensional linear dynamics. Indeed, the Koopman method is closely connected to the Hartman-Grobman theorem in the dynamical systems theory [13] and nonlinear manifold learning in the machine learning community [26], which, however, are studied in a local manner. In [36], the Koopman operator is utilized to provide novel global stability criteria for both hyperbolic equilibria and limit cycles. It was shown that, with a distinct-eigenvalue assumption, the proposed criterion is sufficient and necessary for the asymptotic stability of an equilibrium. However, for many systems the Koopman operator can admit repeated eigenvalues and we are still able to map the dynamics into a linear system globally [20, 22]. Importantly, an attractive feature in Koopman representations is the possibility of data-driven and model-free analysis $[38,40]$. To this end, several techniques have been proposed recently to approximate the Koopman operator by solving least square problems, e.g., dynamic mode decomposition (DMD) [45] and extended (E)DMD [18].

Another way to study nonlinear systems by means of linear methods is contraction analysis. A contracting system has the property that all its trajectories converge to each other, and contraction analysis is based on the study of a nonlinear system in terms of its differential dynamics, which is a linear time-varying (LTV) system along solutions, in this way with linear systems theory being applicable. The key insight is, roughly speaking, that if all solutions are locally stable then all solutions are globally stable. The basic ideas can be traced back to [25], but remarkable utility for problems in control and observer design was noticed much later [27], while connections to Lyapunov methods were elaborated in [11]. Contraction is not just a method for system analysis, but also a powerful constructive tool in many control and learning tasks, see $[32,47,49,57]$ for recent applications in controller design, system identification, online learning and observer design.

In contrast to Koopman operator, which is a single infinitedimensional linear operator, contraction is based on analysis 
of an infinite family (along all feasible solutions) of finitedimensional LTV systems. Due to the similarity - using linear systems theory to analyze nonlinear systems globally - between Koopman and contraction methods, one of the motivations of the paper is to clarify their connections. Despite existing research on Koopman representations for systems with an asymptotically stable equilibrium, it is the authors' opinion that contraction provides a more natural and fundamental form of nonlinear stability to link to Koopman representations than asymptotic stability. The main reason is that, for linear systems, asymptotic stability is a quite strong property, equivalent to various types of stability, e.g., exponential, incremental, and input-to-state stability; contraction shares these strong properties, whereas asymptotic stability of nonlinear systems is relatively weak and sometimes fails to inherit these properties from the lifted linear dynamics of the Koopman operator.

The main contributions of this paper are:

1) Showing the equivalence between contraction and Koopman approaches for stability analysis of nonlinear systems. The stability conditions for autonomous and timevarying nonlinear systems in the Koopman framework imply contraction of the systems; and the converse results also hold true. We give the results for both equilibria and limit cycles with explicit constructions.

2) Establishing the links between control contraction metric (CCM) - a concept to characterize universal stabilizability of nonlinear systems [32] - and stabilizability of the lifted linear systems in the Koopman framework.

3) The constructive solutions in our converse results are obtained from some novel interesting links between the Koopman operator method and Kazantzis-KravarisLuenberger (KKL) observers [3]. The relevant results are of interest on their own.

4) An useful byproduct of the proposed equivalence between Koopman and contraction approaches is that we may learn contraction metrics for stable nonlinear systems from pure trajectory data.

Notation. $\langle\cdot, \cdot\rangle$ and $|\cdot|$ represent standard inner product and norm in the Euclidean space, respectively. All mappings and functions are assumed smooth. Given a matrix $M(x, t)$ and a vector field $f(x)$ with proper dimensions, we define the directional derivative as $\partial_{f} M(x, t):=\sum_{i} \frac{\partial M(x, t)}{\partial x_{i}} f_{i}(x)$ and the operator $\nabla f:=\left(\frac{\partial f}{\partial x}\right)^{\top}$. Given a point $y$, we define a ball $B_{\varepsilon}(y):=\left\{x \in \mathbb{R}^{n}|| x-y \mid<\varepsilon\right\}$. For square matrices $A$ and $B$, the notion $A \prec B$ (or $A \preceq B$ ) indicates $(A-B$ ) negative definite (or semidefinite). We use $\mathbb{R}_{\succ 0}^{m \times m}$ (or $\mathbb{R}_{\succ 0}^{m \times m}$ ) to represent the set of $m \times m$ positive definite (or semidefinite) matrices. A positive definite matrix-valued function $A: \mathbb{R}^{n} \rightarrow$ $\mathbb{R}_{\succ 0}^{m \times m}$ is called uniformly bounded if $a_{1} I \preceq A(x) \preceq a_{2} I, \forall x$ with some $a_{2} \geq a_{1}>0$. We use $\mathbb{C}$ to represent the complex plane, and $\mathbb{C}_{>0}\left(\mathbb{C}_{<0}\right)$ for the open right (left) half-plane. For an open set, $\operatorname{cl}(\cdot)$ denotes its closure. When clear from the context, the arguments of mappings and operators are omitted.

The paper is organized as follows. In Section II we recall some preliminaries on the Koopman operator and contraction analysis. Section III starts with the equivalence between two methods for nonlinear autonomous systems, and the main results are extended to more general time-varying systems in Section IV. Then, it is followed by the relevant results of limit cycles in Section V. Section VI presents some discussions including the relation between the CCM and the Koopman conditions for stabilization problems, as well as the link between the Koopman method and the construction of a KKL observer. Three examples are given in Section VII to illustrate the main results, and the paper is wrapped up with some concluding remarks in Section VIII. A preliminary version of this paper was presented at the conference [55], containing the main results of Section III.

\section{Preliminaries}

We consider both nonlinear autonomous systems of the form

$$
\dot{x}=f(x)
$$

and more general nonlinear time-varying (NLTV) systems

$$
\dot{x}=f(x, t),
$$

with state $x \in \mathbb{R}^{n}$ and the vector field $f \in C^{2}$. The system is assumed complete, i.e., having a unique solution $X(x, t)$ for $t \in[0,+\infty)$ from the initial condition $x$ at $t=0$ for the autonomous system (1), or a solution $X(t ; x, s)$ for the time-varying system (2). In the latter, $X(t ; x, s)$ represents the solution value at time $t$ from the initial condition $x(s)$ at time $s$. In the remainder of the paper, we denote the Jacobian of the function $f$ as $F(x)=\frac{\partial f}{\partial x}(x)$, or $F(x, t)=\frac{\partial f}{\partial x}(x, t)$ for the time-varying case.

\section{A. Contraction Analysis and Control Contraction Metric}

Let us recall the definition of contraction [27], which is given here in its global forms for simplicity.

Definition 1: (contraction) Given a system (2), if there exists a uniformly bounded metric $M(x, t) \in \mathbb{R}_{\succ 0}^{n \times n}$ such that

$$
\dot{M}+\frac{\partial f}{\partial x}(x, t)^{\top} M+M \frac{\partial f}{\partial x}(x, t) \preceq-\rho M, \quad \rho>0,
$$

then we call the system (2) contracting, and $M(x, t)$ is a contraction metric. If the right-hand side of (3) is replaced by " $\prec 0$ ", the given system is asymptotically contracting. $\triangleleft$

Contraction, also known as incremental exponential stability, utilizes the differential behaviour of an infinitesimal displacement $\delta x$ along the flow $X(x, t)$ to characterize the asymptotic behaviour among trajectories of the system (1). To be precise, contraction implies that any two trajectories will exponentially converge to each other, i.e., $\forall\left(x_{a}, x_{b}\right) \in \mathbb{R}^{n} \times \mathbb{R}^{n}$

$$
\left|X\left(x_{a}, t\right)-X\left(x_{b}, t\right)\right| \leq k_{0}\left|x_{a}-x_{b}\right| e^{-\rho t},
$$

for some $k_{0}>0$. For the asymptotic case, it becomes

$$
\left|X\left(x_{a}, t\right)-X\left(x_{b}, t\right)\right| \leq \beta\left(\left|x_{a}-x_{b}\right|, t\right),
$$

in which $\beta(\cdot, \cdot)$ is a class $\mathcal{K} \mathcal{L}$ function.

In $[32,33]$, contraction analysis was extended to the problem of nonlinear control as a constructive method, a benefit of which is its convex representation enabling computational methods for synthesis. The key there is the control contraction metric (CCM), which is related to universal stabilizability - 
a property characterizing if one can find a feedback law to track any feasible trajectories asymptotically. We discuss the problem of stabilization in Section VI-A.

\section{B. Koopman Operator Methods}

The Koopman operator was originally studied for the autonomous system (1). Denote $\mathcal{O}$ as the space of all $C^{2}$-smooth output functions $\varphi: \mathbb{R}^{n} \rightarrow \mathbb{C}$, usually called observables.

Definition 2: (Koopman operator) The Koopman semigroup of operators $U^{t}: \mathcal{O} \rightarrow \mathcal{O}$ associated to the flow $X(x, t)$ for the system (1) is defined by

$$
U^{t}[\varphi]=\varphi \circ X(x, t), \quad \varphi \in \mathcal{O} .
$$

For an observable $\phi_{\lambda} \in \mathcal{O} \backslash\{0\}$, the associated Koopman eigenvalue is defined as the constant $\lambda \in \mathbb{C}$ satisfying

$$
U^{t}\left[\phi_{\lambda}\right]=e^{\lambda t} \phi_{\lambda}
$$

if it exists, and we call $\phi_{\lambda}$ as a Koopman eigenfunction. $\triangleleft$

The operator $U^{t}[\phi]$ has, at a point $x_{A}$, the value which $\phi$ has at the point $X\left(x_{A}, t\right)$ into which $x_{A}$ flows after the lapse of the time $t$ [17]; see Fig. 1. It follows immediately from its definition that the Koopman operator $U^{t}[\cdot]$ is linear.

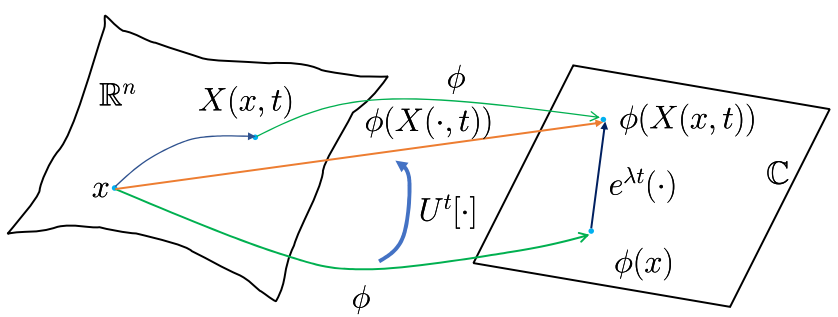

Fig. 1: An illustration of the Koopman operator acting on an observable $\phi$.

Assuming smoothness of observables, the condition (4) may be equivalently formulated as [37]

$$
\left\langle f(x), \frac{\partial \phi_{\lambda}}{\partial x}(x)\right\rangle=\lambda \phi_{\lambda} .
$$

A main result in [36] is that the Koopman operator can be used to verify the global asymptotic stability (GAS) of a hyperbolic equilibrium.

Lemma 1: [36, Proposition 2] Consider the system (1) having a hyperbolic equilibrium $x_{\star}$, with all the eigenvalues of $F\left(x_{\star}\right)$ in $\mathbb{C}_{<0}$. If there exists a mapping $\phi(x):=$ $\left[\phi_{\lambda_{1}}(x), \ldots, \phi_{\lambda_{n}}(x)\right]^{\top}$ such that ${ }^{1}$

1) (distinct eigenvalues) All the Koopman eigenvalues $\lambda_{i}$ are distinct and $\phi_{\lambda_{i}} \in C^{1}$ with $\nabla \phi_{\lambda_{i}}\left(x_{\star}\right) \neq 0$ for $i=$ $1, \ldots, n$;

2) (stability) $\lambda_{i} \in \mathbb{C}_{<0}$ satisfy the PDEs (5), and are the eigenvalues of $F\left(x_{\star}\right)$.

Then, the equilibrium $x_{\star}$ is GAS.

The PDEs in the condition 2) immerse the autonomous system (1) into the linear time-invariant (LTI) dynamics

$$
\dot{z}=\Lambda z, \quad z(0)=\phi(x(0))
$$

with $\Lambda=\operatorname{diag}\left(\lambda_{1}, \ldots, \lambda_{n}\right)$ via the change of coordinate $x \mapsto$ $z=\phi(x)$. By the condition $\lambda_{i} \in \mathbb{C}_{<0}$, this linear system is globally exponentially stable (GES) at the origin.

\footnotetext{
${ }^{1}$ The following conditions are also necessary for the GAS of $x_{\star}$.
}

\section{Main Results for Autonomous Systems}

In this section, we start with the time-invariant system (1), showing the equivalence between the Koopman and contraction approaches.

\section{A. Koopman Implies Contraction for Stability of Autonomous Systems}

In Lemma 1, the second assumption requires distinct eigenvalues, requiring the LTI system (6) diagonalizable, which is somewhat restrictive ${ }^{2}$. Before presenting our first result, we slightly extend Lemma 1 as follows.

Proposition 1: Consider the system (1) with a hyperbolic equilibrium $x_{\star}$. If there exists a mapping $\phi(x):=$ $\left[\phi_{1}(x), \ldots, \phi_{N}(x)\right]^{\top}$ with $(N-n) \in \mathbb{N} \cup\{\infty\}$ such that

C1 (immersion) For a finite $N, \Phi(x):=\frac{\partial \phi}{\partial x}(x)$ is full column rank; if $N$ is infinite, $\phi(x)$ is assumed to be rank- $n$ countably-infinite, i.e., there are $\ell \geq n$ elements (denoted as $\left.\phi_{k_{j}}, j=1, \ldots, \ell\right)$ of $\phi$ such that $\operatorname{rank}\{\nabla \tilde{\phi}(x)\}=n$, with $\phi:=\operatorname{col}\left(\phi_{k_{1}}, \ldots, \phi_{k_{\ell}}\right)$.

C2 (stability) There exists exponentially stable $A$ verifying

$$
\frac{\partial \phi}{\partial x}(x) f(x)=A \phi(x) .
$$

Then, the equilibrium $x_{\star}$ is GAS.

Proof: We write the dynamics in the $z$-coordinate as

$$
\dot{z}=A z, \quad z(0)=\phi(x(0)) .
$$

From the assumption C2, $z \rightarrow 0$ exponentially as $t \rightarrow \infty$. Since $x_{\star}$ is an equilibrium, we have $f\left(x_{\star}\right)=0$, and invoking (7) yields $\phi\left(x_{\star}\right)=0$. From the immersion condition $\mathbf{C 1}$, if $N \in \mathbb{N}$, then $\phi: \mathbb{R}^{n} \rightarrow \mathbb{R}^{N}$ is locally injective around $x_{\star}$, thus there is a class $\mathcal{K}$ function $\beta$ such that

$$
\left|x_{a}-x_{b}\right| \leq \beta\left(\left|\phi\left(x_{a}\right)-\phi\left(x_{b}\right)\right|\right), \forall\left(x_{a}, x_{b}\right) \in B_{\varepsilon}\left(x_{\star}\right)^{2}
$$

with $\varepsilon>0$ sufficiently small. By substituting $x_{b}$ as $x_{\star}$ and $x_{a}$ as $X(x, t)$ with $x \in B_{\varepsilon}\left(x_{\star}\right)$, we conclude that $x_{\star}$ is locally attractive for the system (1). In terms of hyperbolicity of the equilibrium $x_{\star}$, the Jacobian $F\left(x_{\star}\right)$ is Hurwitz.

Since $F\left(x_{\star}\right)$ is Hurwitz and the condition C1 holds for all $x \in \mathbb{R}^{n}$, as well as invoking the fact that basin of attraction is open [44, Proposition 5.44], the preimage $\phi^{-1}(0)$ only contains a single isolated equilibrium point. Hence, the function $\phi$ is injective on the entire basin of attraction of $x_{\star}$. Due to the exponential stability of the $z$-dynamics, there always exists a moment $t_{\star}$ such that $x(t) \in B_{\varepsilon}\left(x_{\star}\right)$ for $t \geq t_{\star}$ from any initial condition. Then, for all $x(0) \in \mathbb{R}^{n}$ we have

$$
\begin{aligned}
\left|x(t)-x_{\star}\right| & \leq \beta\left(\left|e^{A t} \phi(x(0))-\phi\left(x_{\star}\right)\right|\right) \\
& \leq \beta\left(\left|e^{A t} \phi(x(0))\right|\right), \quad \forall t \geq t_{\star},
\end{aligned}
$$

which can be upper bounded by a $\mathcal{K} \mathcal{L}$ function of $\left|x(0)-x_{\star}\right|$ and $t$, thus obtaining the GAS. If $N$ is infinite, we may use $\tilde{\phi}$ to do a similar analysis.

Note that the mapping $\phi$ in Proposition 1 is a little different from the Koopman eigenfunctions, since it does not require the diagonalizability of $A$. Here we call $\phi(x)$ the Koopman

\footnotetext{
${ }^{2}$ This could be relaxed by considering "generalized eigenfunctions" in [39].
} 
mapping, which is usually referred as semiconjugacy in the dynamical systems literature. The connection between Proposition 1 and Lemma 1 is established by the PDEs (5) and (7). If the matrix $A$ is diagonalizable with $A=T^{-1} \Lambda T$ and $N \in \mathbb{N}$, then $T \phi(x)$ is a set of Koopman eigenfunctions associated with the eigenvalues $\lambda_{1}, \ldots, \lambda_{N}$. Note that the mapping $\phi(x)$ identified in C1-C2 is not unique, in contrast to the principal eigenfunctions used in Lemma 1.

Remark 1: The condition $\mathbf{C 1}$ implies the existence of a local inverse at each point, but not necessarily global, i.e., it corresponds to an immersion rather than an embedding. The gap is that $\phi$ may be not proper in many cases due to $N \geq n$. In some cases we may want the stronger condition that $\phi$ has a left inverse $\phi^{\mathrm{L}}$ with $\phi^{\mathrm{L}}(\phi(x))=x$. Note that both trivially hold if $\phi$ contains the original system states, i.e. $\phi(x)=\operatorname{col}\left(x, \phi^{\prime}(x)\right)$ for some $\phi^{\prime}$. The existence of a Koopman mapping $\phi(x)$ which satisfies the immersion condition is closely related to having a rectifiable dynamics $[12,19]$ such that it yields a local inverse.

We are now in position to present the first main result of the paper.

Theorem 1: Assuming that there exists a $C^{2}$ Koopman mapping $\phi$ satisfying C1-C2 and $\Phi^{\top} \Phi$ is uniformly bounded, then the system (1) is contracting with a contraction metric

$$
M(x)=\Phi(x)^{\top} P \Phi(x),
$$

where $P$ is the solution to $A^{\top} P+P A=-I{ }^{3}$

Proof: If there exists a Koopman mapping $\phi$ satisfying C1-C2, invoking that $A$ is exponentially stable, then there exists $P=P^{\top} \succ 0$ satisfying the Lyapunov equation ${ }^{4}$

$$
A^{\top} P+P A=-I \text {. }
$$

From the assumptions, the mapping $\phi$ satisfies the PDE (7). Now, we calculate the partial derivative with respect to $x$ in each side, and denote $\Phi(x):=\frac{\partial \phi}{\partial x}(x)$, yielding

$$
\begin{aligned}
\partial_{f} \Phi(x)+\frac{\partial \phi}{\partial x}(x) \frac{\partial f}{\partial x}(x) & =A \Phi(x) \\
\Longrightarrow \quad \dot{\Phi}(x)+\Phi(x) F(x) & =A \Phi(x) .
\end{aligned}
$$

From the Lyapunov equation, we have

$$
\Phi^{\top}\left(A^{\top} P+P A\right) \Phi=-\Phi^{\top} \Phi \prec 0,
$$

where we have used the full rank assumption of $\Phi(x)$ in C1, and the fact that $\Phi(x)$ is a tall matrix. On the other hand,

$$
\begin{array}{ll} 
& \Phi^{\top}\left(A^{\top} P+P A\right) \Phi \\
\stackrel{(10)}{=} & \Phi^{\top} P \dot{\Phi}+\dot{\Phi}^{\top} P \Phi+\Phi^{\top} P \Phi F+F^{\top} \Phi^{\top} P \Phi \\
\stackrel{(9)}{=} & \dot{M}+F^{\top} M+M F \\
\stackrel{(11)}{=} & -\Phi^{\top} \Phi .
\end{array}
$$

Applying

$$
\Phi^{\top} \Phi \succeq \frac{1}{\lambda_{\max }\{P\}} \Phi^{\top} P \Phi
$$

\footnotetext{
${ }^{3}$ For infinite $N$, the Lyapunov equation becomes $\langle A z, P z\rangle+\langle P z, A z\rangle=$ $-\langle z, z\rangle, z \in D(A)$ with $D(A)$ the domain of the infinitesimal generator $A$.

${ }^{4}$ This is also true for infinite-dimensional systems [9, Thm. 5.13].
}

with the largest eigenvalue $\lambda_{\max }\{P\}$, we then have

$$
\dot{M}+F^{\top} M+M F \prec-\frac{1}{\lambda_{\max }\{P\}} M .
$$

It implies the contraction of the nonlinear system (1).

Remark 2: The above proof boils down to the application of contraction of the linear lifting dynamics $\dot{z}=A z$. Though it is well-known that incremental stability is intrinsic [11], the special point in the proof relies on the transformation $\phi$ : $x \mapsto z$ being an immersion rather than a diffeomorphism. We underline that the immersion is guaranteed by the full rank condition $\mathbf{C 1}$, which prevents $\phi$ from mapping another point $x^{\prime}$ in a small neighborhood of the equilibrium $x_{\star}$ to the origin $z=0$ in the lifted coordinate. Furthermore, the full rank condition ensures that the contraction metric $M$ in (9) is positive definite; see Definition 1.

Remark 3: It was shown in [35] that the existence of a set of eigenfunctions $\phi_{\lambda_{i}}$ is related to a "contracting metric"

$$
d\left(x_{1}, x_{2}\right)=\left(\sum_{i=1}^{N}\left|\phi_{\lambda_{i}}\left(x_{1}\right)-\phi_{\lambda_{i}}\left(x_{2}\right)\right|^{p}\right)^{\frac{1}{p}}
$$

with integer $p \geq 1$, which follows the set stability framework to study incremental stability [2]. In the past decade, there has been more attention on the differential framework to analyze incremental stability, and our main results follow this line. It is clear that the obtained $M=\Phi^{\top} P \Phi$ is a Riemannian metric defined on tangent bundle, whereas (12) is defined in state space. The former enjoys attractive computational convenience in many settings $[32,34]$.

Remark 4: The stability criterion in Proposition 1 requires finding a function $\phi$ and a matrix $A$ satisfying C1-C2 simultaneously, making it non-trivial to verify. In [36], the authors provide numerical methods for the criterion in Lemma 1 in terms of Taylor expansion or Bernstein polynomials. In the next section, we will show that contraction is also sufficient for C1-C2. As a result, we may use the convex condition (3) to verify the conditions C1-C2 indirectly, e.g. by means of convex optimization and sum-of-squares $[32,34]$.

\section{B. Contraction Implies Koopman for Stability of Autonomous Systems}

In this subsection, we prove the converse of Theorem 1.

Theorem 2: Consider the autonomous system (1), which is contracting with the metric $M(x)$ in a compact set $\operatorname{cl}(\mathcal{X})$. Then, there always exists always a $C^{1}$ Koopman mapping $\phi$ satisfying C1-C2 with $N \in \mathbb{N}$.

Proof: For a system forward invariant in a closed set, if it is autonomous and contracting, we can prove that the system admits a unique GES equilibrium $x_{\star}$, i.e. $f\left(x_{\star}\right)=0$, using the well-known Banach fixed point theorem; see for example [14]. (It holds true for the case that the set $\mathcal{X}$ is exactly $\mathbb{R}^{n}$, which is both closed and open.) Since we can always assign the equilibrium by a linear coordinate change $x \mapsto\left(x-x_{\star}\right)$, without loss of generality, we assume the equilibrium $x_{\star}$ is at the origin.

The system dynamics (1) can be written as

$$
\dot{x}=F_{\star} x-H(x), \quad F_{\star}:=F\left(x_{\star}\right)
$$


with the high-order remainder term

$$
H(x):=-f(x)+F_{\star} x .
$$

From the contraction assumption, we have

$$
\partial_{f} M(x)+F(x)^{\top} M(x)+M(x) F(x) \leq-\gamma M(x)
$$

with $\gamma>0$ along all feasible solutions, and the metric $M(x)$ uniformly bounded. After substituting the particular solution $X\left(x_{\star}, t\right)=x_{\star}, \forall t \geq 0$, we obtain

$$
F_{\star}^{\top} M\left(x_{\star}\right)+M\left(x_{\star}\right) F_{\star} \leq-\gamma M\left(x_{\star}\right),
$$

in which we have used $f\left(x_{\star}\right)=0$, thus $\dot{M}\left(X\left(x_{\star}, t\right)\right)=0$. The above inequality implies that $F_{\star}$ is Hurwitz. Hence, $x_{\star}$ is a hyperbolic equilibrium.

We consider the case $N=n$, and parameterize $\phi$ as

$$
\phi(x)=x+T(x)
$$

with a smooth function $T$ to be searched for. Substituting (13) into the PDE (7) and fixing $A=F_{\star}$, we have

$$
\begin{array}{cc} 
& {\left[I+\frac{\partial T}{\partial x}(x)\right] f(x)=A(x+T(x))} \\
\Longrightarrow \quad F_{\star} x-H(x)+\frac{\partial T}{\partial x}(x) f(x)=A T(x)+A x \\
\stackrel{A=F_{\star}}{\Longrightarrow} \frac{\partial T}{\partial x}(x) f(x)=A T(x)+H(x) .
\end{array}
$$

Note that we have already shown $A=F_{\star}$ is Hurwitz from the contraction assumption. It is interesting to observe that the last PDE in (14) is identical to the one appearing in the KKL observer [16]. That is, finding a mapping $\phi$ verifying C2 is equivalent to solving the PDE in KKL observers with respect to the new mapping $T$. $^{5}$

The remainder of the proof follows some constructive solutions to KKL observers [16], in which it is assumed that the solution $X(x, t)$ does not blow-up in finite backward time. When this additional assumption does not hold, we may still continue the analysis by considering the modified dynamics

$$
\dot{x}=\rho(x) f(x)
$$

with an arbitrary $C^{\infty}$ function $\rho: \mathbb{R}^{n} \rightarrow \mathbb{R}$ satisfying

$$
\rho(x)= \begin{cases}1, & \text { if } \quad x \in \mathrm{cl}(\mathcal{X}) \\ 0, & \text { if } \quad x \notin \mathcal{X}^{\prime}\end{cases}
$$

for some $\operatorname{cl}(\mathcal{X}) \subset \mathcal{X}^{\prime} \subset \mathbb{R}^{n}$. Such a modification makes the above backward assumption always hold. Note that we may select the compact set $c l(\mathcal{X})$ arbitrarily large. Let us denote the solution of the modified dynamics (15) as $X(x, t)$.

As shown in [1], the PDE (14) has a feasible solution

$$
T(X(x, t))=e^{F_{\star} t} T(x)+\int_{0}^{t} e^{F_{\star}(t-s)} H(X(x, s)) d s
$$

if it is well-posed. Since $F_{\star}$ is Hurwitz, it has a solution when $t \rightarrow+\infty$, and we select

$$
T(x)=\int_{0}^{+\infty} \exp \left(F_{\star} s\right) H(\breve{X}(x,-s)) d s .
$$

\footnotetext{
${ }^{5}$ We refer the interested reader to Section VI-B for a brief introduction to KKL observers.
}

Therefore, by selecting

$$
\phi^{0}(x)=x+T(x)
$$

with the $C^{1}$ function $T$ defined in (17), we get a feasible solution in $\operatorname{cl}(\mathcal{X})$ to the PDE (7).

The remainder is to verify the full rank condition $\mathbf{C 1}$ by redesigning the function $\phi^{0}(x)$. Combining the facts

$$
T\left(x_{\star}\right)=0, \quad \nabla T\left(x_{\star}\right)=0
$$

and

$$
\frac{\partial \phi^{0}}{\partial x}(x)=I_{n}+\frac{\partial T}{\partial x}(x),
$$

we conclude from the continuity that there always exists a small parameter $\varepsilon>0$ such that the mapping $\phi^{0}(x)$ is an injection in the neighborhood $B_{\varepsilon}\left(x_{\star}\right)$ of the equilibrium $x_{\star}$. However, it may be not true for the entire $\mathbb{R}^{n}$. Motivated by [22, Thm. 2.3] and [54], we need to modify the mapping $\phi^{0}(x)$. Since we may, via the change of coordinate $z=\phi^{0}(x)$, transform the dynamics into $\dot{z}=F_{\star} z$, we now write its flow as $Z(z, t)=e^{A t} z$ from the initial condition $z \in \mathbb{R}^{n}$, and the dynamics is complete for $t \in(-\infty,+\infty)$. Note that, for the above construction, we have $Z(z, t)=\phi^{0}(X(x, t))$ for any $t$ and $z=\phi^{0}(x)$. We may derive the solution of $Z(\cdot)$ at $t_{x}>0$ in the lifted linear $z$-coordinate as

$$
Z\left(z, t_{x}\right)=e^{A t_{x}} z=e^{A t_{x}} \phi^{0}(x) .
$$

On the other hand, we may calculate its solution using the flow in the original $x$-coordinate, and then lifting it to the $z$-coordinate, i.e.

$$
Z\left(z, t_{x}\right)=\phi^{0}\left(X\left(x, t_{x}\right)\right)
$$

By combining (18) and (19), we have

$$
\phi^{0}(x)=e^{-A t_{x}} \phi^{0}\left(X\left(x, t_{x}\right)\right) .
$$

Hence, re-defining the Koopman mapping as

$$
\phi(x):=e^{-A t_{x}} \phi^{0}\left(X\left(x, t_{x}\right)\right),
$$

which still satisfies $\mathbf{C 2}$ for any $t_{x}>0$. Regarding $\mathbf{C 1}$, we have

$$
\Phi(x)=e^{-A t_{x}} \frac{\partial \phi^{0}}{\partial x}\left(X\left(x, t_{x}\right)\right) \frac{\partial X}{\partial x}\left(x, t_{x}\right) .
$$

For a very large $t_{x}>0, X\left(x, t_{x}\right)$ is in a small neighborhood of the origin, and thus both $\nabla \phi^{0}\left(X\left(x, t_{x}\right)\right)$ and $\frac{\partial X}{\partial x}\left(x, t_{x}\right)$ are full rank. Thus, the Jacobian $\Phi(x)$ is full rank globally.

Remark 5: It is shown in [36] that the assumptions in Lemma 1 are also necessary for GAS of autonomous systems, similar to the converse result in Theorem 2. However, the difference is clear that Lemma 1 requires a distinct-eigenvalue assumption, making it somewhat restrictive, and instead, we impose an immersion condition. As a consequence, the PDEs in Lemma 1 and $\mathbf{C 2}$ are slightly different depending on whether the system matrix $A$ is diagonalizable.

Remark 6: In the above proof, we verify the condition $\mathbf{C 2}$ by studying the flows in both the $x$ - and $z$-coordinates. 
Indeed, we may verify directly the PDE (7) for the re-designed mapping $\phi(x)$ in (21). To be precise, we have

$$
\begin{aligned}
\frac{\partial \phi}{\partial x}(x) f(x) & =e^{-A t_{x}} \frac{\partial \phi^{0}}{\partial x}\left(X\left(x, t_{x}\right)\right) \frac{d X}{d t}\left(x, t_{x}\right) \\
& =e^{-A t_{x}} \frac{\partial \phi^{0}}{\partial x}\left(X\left(x, t_{x}\right)\right) f\left(X\left(x, t_{x}\right)\right) \\
& =e^{-A t_{x}} A \phi^{0}\left(X\left(x, t_{x}\right)\right) \\
& =A e^{-A t_{x}} \phi^{0}\left(X\left(x, t_{x}\right)\right) \\
& =A \phi(x) .
\end{aligned}
$$

Remark 7: In both Theorem 1 and its converse claim Theorem 2 - we consider the stronger condition of full rank of $\Phi(x)$ than those used in the previous results, showing the equivalence of Koopman methods to contraction analysis, not just the stability of the origin. This fact will be further elaborated for time-varying systems.

Remark 8: It should be underscored that there is a gap on smoothness between Theorem 1 and its converse result. The standing assumption in Theorem 1 is that $\phi \in C^{2}$; however, in the conservse result in Theorem 2 our construction of $\phi$ in (21) is only $C^{1}$. In [20] it is shown that $\phi \in C^{2}$ defined on the entire domain of attraction exists by imposing the stronger assumption of $f \in C^{\infty}$ and the nonresonance of $F\left(x_{\star}\right)$. Note that the nonresonance condition is also used in [16, Eq. (5)] to study the solution to the PDE (14) in the context of KKL observers.

\section{Main Results for Time-Varying Systems}

In this section, we extend the results in Section III from autonomous systems to NLTV systems.

\section{A. The Koopman Operator for NLTV systems}

The Koopman operator was originally defined for autonomous systems, and its definition was extended to NLTV systems in [28] and the controlled system with inputs in [42]. Consider $\mathcal{O}$ as the Hilbert space of all $C^{2}$-smooth time-varying observables $\varphi: \mathbb{R}^{n} \times \mathbb{R} \rightarrow \mathbb{C}$ with the usual inner product. In consistent with [28], we define the non-autonomous Koopman operator for the NLTV system (2) with two parameters $(s, t)$ as

$$
U^{(t, s)}[\varphi(x, s)]:=\varphi(X(t ; x, s), t),
$$

which is able to characterize the time-varying property of the system; see Fig. 2. In the non-autonomous Koopman operator, we also allow the observables being time-varying, which is consistent with Definition 2. Note that for such an extension, the Koopman operator - defined as a semi-group - is still linear.

In a similar way, we define the Koopman eigenfunctions $\phi_{\lambda}(x, t)$ and Koopman eigenvalues $\lambda: \mathbb{R} \rightarrow \mathbb{C}$ by

$$
a(t, s)=\int_{s}^{t} \lambda(\tau) d \tau,
$$

and then

$$
U^{(t, s)}\left[\phi_{\lambda}\right]=e^{a(t, s)} \phi_{\lambda},
$$

which, for the autonomous systems, clearly degenerates into $U^{(t, s)}\left[\phi_{\lambda}\right]=e^{\lambda(t-s)} \phi_{\lambda}$. It is compatible with the autonomous definition selecting $s=0$.

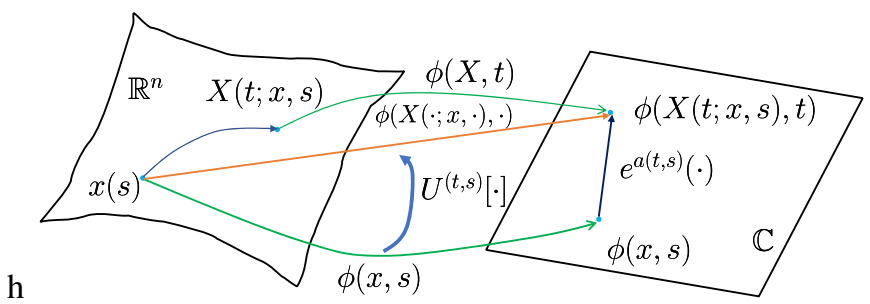

Fig. 2: Non-autonomous Koopman operator acting on a timevarying observable $\phi(x, t)$

The infinitesimal generator $A_{U}^{s}$ of the non-autonomous Koopman operator $U^{(t, s)}[\cdot]$ is defined as [28]

$$
\begin{aligned}
A_{U}^{s} \varphi(x, t) & :=\lim _{t \rightarrow s} \frac{U^{(t, s)}[\varphi(x, s)]-\varphi(x, s)}{t-s} \\
& =\lim _{t \rightarrow s} \frac{\varphi(X(t ; x, s), t)-\varphi(x, s)}{t-s} \\
& =\left.\overbrace{\varphi(x, t)}\right|_{t=s},
\end{aligned}
$$

assuming that the observable $\varphi$ is smooth. Now considering the eigenfunction $\phi_{\lambda}(x, t)$ and invoking (23), we have

$$
\begin{aligned}
\overparen{\phi_{\lambda}(x, t)} & =\frac{\partial \phi_{\lambda}}{\partial t}+\left\langle f(x, t), \frac{\partial \phi_{\lambda}}{\partial x}\right\rangle \\
& =\lim _{t \rightarrow s} \frac{e^{a(t, s)}-1}{t-s} \lim _{t \rightarrow s} \phi_{\lambda}(t, s) \\
& =\left.\frac{d a(s, t)}{d t}\right|_{s=t} \phi_{\lambda}(x, t) \\
& =\lambda(t) \phi_{\lambda}(x, t) .
\end{aligned}
$$

Equivalently, the Koopman eigenfunction $\phi_{\lambda} \in \mathcal{O}$ and the eigenvalue $\lambda \in \mathbb{C}$ satisfies the PDE

$$
\frac{\partial \phi_{\lambda}}{\partial t}+\left\langle f(x, t), \frac{\partial \phi_{\lambda}}{\partial x}\right\rangle=\lambda \phi_{\lambda} .
$$

If we are able to find a family of eigenfunctions $\phi(x, t):=$ $\left[\phi_{\lambda_{1}}, \ldots, \phi_{\lambda_{N}}\right]^{\top}$ with $N \geq n$, then the given NLTV system (2) can be immersed into an LTV system ${ }^{6}$

$$
\dot{z}=\Lambda(t) z
$$

with $z=\phi(x, t)$ and $\Lambda(t):=\operatorname{diag}\left(\lambda_{1}(t), \ldots, \lambda_{N}(t)\right)$.

Following Subsection III-A, we define the (time-varying) Koopman mapping $\phi(x, t)$ to remove the diagonalizable constraint, which is required to satisfy the PDE

$$
\frac{\partial \phi}{\partial t}(x, t)+\frac{\partial \phi}{\partial x}(x, t) f(x, t)=A(t) \phi(x, t)
$$

with a time-varying matrix $A(t)$ Lyapunov stable, i.e., for any positive definite matrix $Q$ there exists a positive definite matrix $P(t)$ satisfying [50]

$$
\dot{P}(t)+A^{\top}(t) P(t)+P(t) A(t)+Q=0 .
$$

If $P(t)$ is uniformly bounded, the lifted LTV system

$$
\dot{z}=A(t) z, \quad z(0)=\phi(x(0), 0)
$$

is uniformly exponentially stable (UES).

${ }^{6}$ It should be underlined that, in many cases, we are still able to find constant Koopman eigenfunctions for some classes of time-varying systems (2). As a result, the obtained lifted linear systems are time-invariant. 


\section{B. Koopman Implies Contraction for NLTV Systems}

In this subsection, we show that a variant of Theorem 1 holds for time-varying systems.

Theorem 3: Consider the NLTV system (2), and assume that there exists a $C^{2}$-Koopman mapping $\phi(x, t) \in \mathbb{R}^{N}$ satisfying

C1' (immersion) For $N \in \mathbb{N}$,

$$
\operatorname{rank}\left\{\frac{\partial \phi(x, t)}{\partial x}\right\}=n, \quad \forall x \in \mathbb{R}^{n}, t \in \mathbb{R}_{\geq 0} .
$$

C2 $^{\prime}$ (stability) the existence of an UES and bounded $A(t)$ verifying the PDE (25).

If $\left[\frac{\partial \phi(x, t)}{\partial x}\right]^{\top} \frac{\partial \phi(x, t)}{\partial x}$ is uniformly bounded, then the system is contracting.

Proof: We define the partial derivative $\Phi(x, t):=$ $\frac{\partial \phi}{\partial x}(x, t)$. For the PDE (25), by calculating the partial derivative with respect to $x$, we obtain

$$
\frac{\partial^{2} \phi}{\partial x \partial t}+\partial_{f} \Phi+\frac{\partial \phi}{\partial x} \frac{\partial f}{\partial x}=A \frac{\partial \phi}{\partial x} .
$$

Since $\phi \in C^{2}$, we have $\frac{\partial^{2} \phi}{\partial x \partial t}(x, t)=\frac{\partial^{2} \phi}{\partial t \partial x}(x, t)$ then

$$
\begin{aligned}
& \frac{\partial^{2} \phi}{\partial t \partial x}+\partial_{f} \Phi+\frac{\partial \phi}{\partial x} \frac{\partial f}{\partial x}=A \frac{\partial \phi}{\partial x} \\
\Longleftrightarrow & \frac{\partial \Phi}{\partial t}+\partial_{f} \Phi+\Phi F=A \Phi \\
\Longleftrightarrow & \overparen{\Phi(x, t)}+\Phi(x, t) F(x, t)=A(t) \Phi(x, t) .
\end{aligned}
$$

From the UES of $A(t)$, there exists $P(t) \succ 0$ satisfying the differential Lyapunov inequality [43, Thm. 7.8]

$$
\dot{P}(t)+A^{\top}(t) P(t)+P(t) A(t) \preceq-k I_{n}
$$

and the uniform boundedness

$$
p_{1} I_{n} \preceq P(t) \preceq p_{2} I_{n}, \quad \forall t \geq 0
$$

for some $k>0$ and $p_{2}>p_{1}>0$.

Choose the contraction metric

$$
M(x, t)=\Phi(x, t)^{\top} P(t) \Phi(x, t),
$$

in which $P(t) \succ 0$ is the solution of the differential Lyapunov inequality (29). Then, it yields

$$
\begin{aligned}
& \dot{M}+F^{\top} M+M F \\
= & \dot{\Phi}^{\top} P \Phi+\Phi^{\top} P \dot{\Phi}+\Phi^{\top} \dot{P} \Phi+F^{\top} \Phi^{\top} P \Phi+\Phi^{\top} P \Phi F \\
= & \Phi^{\top}\left(A^{\top} P+P A+\dot{P}\right) \Phi \\
\preceq & -k I_{n} .
\end{aligned}
$$

Since both $P(t)$ and $\Phi^{\top} \Phi$ are uniformly bounded, the matrix $M(x, t)$ defined above is qualified as a uniformly bounded contraction metric. It completes the proof.

The above result is not surprising, since contraction analysis was originally tailored for time-varying systems. From Theorems 1 and 3, the Koopman-based stability analysis may be roughly viewed as an alternative formulation of contraction analysis.

\section{A General Converse Result}

In this subsection, we study the general converse results for nonlinear contracting systems, that is, the stability of infinitesimal generator of the Koopman operator $U^{(t, s)}$ for a class of time-varying observables.

Proposition 2: If the NLTV system (2) is contracting with bounded trajectories, then for the class of time-varying observables $\phi: \mathbb{R}^{n} \times \mathbb{R}_{+} \rightarrow \mathbb{R}$, parameterized as

$$
\phi(x, t)=\varphi(x, t)-\varphi\left(X\left(t ; x_{a}, 0\right), t\right)
$$

with any $C^{1}$-smooth function $\varphi$ and any point $x_{a} \in \mathbb{R}^{n}$, the associated infinitesimal generator of $U^{(t, s)}$, i.e., $A_{U}^{s} \phi=$ $\lim _{t \rightarrow s}\left(U^{(t, s)}[\phi]-\phi\right) /(t-s)$ guarantees that there exists a positive operator $P$ satisfying

$$
\left\langle A_{U}^{s} z, P z\right\rangle+\left\langle P z, A_{U}^{s} z\right\rangle=-\langle z, z\rangle, z \in D\left(A_{U}^{s}\right) .
$$

Proof: For the system (2), we denote the output of the Koopman operator from $s$ to $t$ as

$$
g(x, t, s):=U^{(t, s)}[\phi(x, s)]
$$

for given $t \geq s \geq 0$, with the initial condition constraint $g(x, s, s)=\phi(x, s)$. Invoking the smoothness assumption and according to [23, Thm. 7.5.1], the infinitesimal generator is equivalently defined as

$$
\begin{aligned}
\frac{\partial}{\partial t} g(x, t, s) & =\frac{\partial}{\partial t} U^{(t, s)}[\phi(x, s)] \\
& =\frac{\partial}{\partial t} \phi(X(t ; x, s), t) \\
& =A_{U}^{s} g(x, t, s),
\end{aligned}
$$

in which the operator $A_{U}^{s}$ is infinite dimensional.

We may verify that there is a linear operator $T(t, s)$ guaranteeing

$$
g(x, t, s)=T(t, s)[g(x, s, s)]
$$

with $T(t, s)=U^{(t, s)}$ invoking $g(x, s, s)=\phi(x, s)$ and the definition of the non-autonomous Koopman operator in (22). Considering the smoothness of $\psi$, the induced norm on the Hilbert space $\mathcal{O}$ and the continuity of the function $X\left(t ; x_{a}, 0\right)$ which is generated by the flow a dynamical system, we obtain that $T(t, s)$ is a strongly continuous semigroup on the Hilbert space $\mathcal{O}$ [9, Def. 2.1.2].

For the class of observables defined in (30), invoking the contraction of the system, the output $g(x, t, s)$ of the Koopman operator exponentially converges to zero as $t \rightarrow \infty$, i.e., $|g(x, t, s)| \leq k e^{-\alpha(t-s)}|g(x, s, s)|$ for any $x \in \mathbb{R}^{n}$ and some $k, \alpha>0$. In the associated abstract Cauchy problem, the semigroup is exponentially stable. According to [9, Thm. 5.1.3], we obtain the Lyapunov equation (31) for the infinitesimal generator $A_{U}^{s}$.

In the above we consider the infinite-dimensional case with $A_{U}^{s}$ a stable operator. This general result, however, is difficult to use in practice. We are more interested in a finitedimensional $A_{U}^{s}$. Such a problem will be studied in the next subsection under different assumptions.

\footnotetext{
${ }^{7}$ Here, $g(x, t, s)$ is thought as a function of isolated coordinates $x, t$ and $s$ that evolve independently, see for example [23, Remark 7.5.2].
} 


\section{Contraction Implies Koopman for NLTV Systems}

Following the above subsection, we first consider the case to embed a contracting NLTV system into an LTV system.

Theorem 4: Consider the NLTV system (2) with an equilibrium $x_{\star}$ at the origin, and assume that the system is contracting with a smooth time-varying metric $M(x, t)$ in a compact set $\operatorname{cl}(\mathcal{X})$. Then, there always exists a Koopman mapping $\phi(x, t)$ satisfying $\mathbf{C 1}^{\prime}$ and $\mathbf{C 2}^{\prime}$. Further, the claims still hold true if the systems state is bounded but removing the assumption of the existence of an equilibrium $x_{\star}$.

Proof: From the contraction of the system (2), we know the UES of the LTV system

$$
\delta \dot{x}=\frac{\partial f}{\partial x}(X(t ; x, s), t) \delta x, \quad t \geq s
$$

with the infinitesimal displacement $\delta x \in T \mathbb{R}^{n}$, any $s \geq 0$ and

$$
\dot{M}+\frac{\partial f^{\top}}{\partial x} M+M \frac{\partial f}{\partial x} \preceq-\rho M, \quad \rho>0 .
$$

As a particular solution from the equilibrium $x_{\star}$, we conclude the UES of

$$
A(t):=\frac{\partial f}{\partial x}\left(x_{\star}, t\right),
$$

i.e., satisfying the differential Lyapunov inequality (29) with

$$
P(t)=M\left(X\left(t ; x_{\star}, 0\right), t\right)=M\left(x_{\star}, t\right) .
$$

Now let us parameterize the Koopman transformation as

$$
\phi(x, t)=x+T(x, t),
$$

with a $C^{1}$-mapping $T: \mathbb{R}^{n} \rightarrow \mathbb{R}_{\geq 0}$ to search, and define

$$
H(x, t)=-f(x, t)+A(t) .
$$

Then, the PDE (25) in the non-autonomous Koopman approach becomes

$$
\frac{\partial T}{\partial x}(x, t)+\frac{\partial T}{\partial x}(x, t) f(x, t)=A(t) T(x, t)+H(x, t),
$$

with a stable $A(t)$. We denote the associated state transition matrix of $A(t)$ as $\Omega(t, s)$, and we have

$$
z(t)=\Omega(t, s) z(s)
$$

for the LTV system (27). It is a function of $A(t)$, but most time it is impossible to write its explicit formula, and we may numerically obtain from

$$
\dot{\Phi}_{A}(t)=A(t) \Phi_{A}(t), \quad \Phi_{A}(0)=I_{n}
$$

with $\Omega(t, s)=\Phi_{A}(t) \Phi_{A}(s)^{-1}$.

The next step is to show the mapping

$$
T^{0}(x, t)=\int_{0}^{t} \Omega(t, s) H(X(s ; x, t), s) d s
$$

is a feasible solution to the PDE (32) for $x \in \mathcal{X}{ }^{8}$ To this end, motivated by [4], we note that for any $x \in \mathbb{R}^{n}, t \in[0,+\infty)$ and any $\tau$, we have

$$
X(s ; X(t+\tau, x, t), t+\tau)=X(s ; x, t),
$$

\footnotetext{
${ }^{8}$ Here, we assume that for the NLTV system (2) there is no backward finite-time escaping. Otherwise, we may adopt the modification in (15).
}

and then

$$
\begin{aligned}
& T^{0}(X(t+\tau ; x, t), t+\tau) \\
= & \int_{0}^{t+\tau} \Omega(t+\tau, s) H(X(s ; x, t), s) d s \\
= & \int_{0}^{t+\tau} \Omega(t+\tau, t) \Omega(t, s) H(X(s ; x, t), s) d s \\
= & \Omega(t+\tau, t) \int_{0}^{t+\tau} \Omega(t, s) H(X(s ; x, t), s) d s \\
= & \Omega(t+\tau, t) T^{0}(x, t) \\
& \quad+\Omega(t+\tau, t) \int_{t}^{t+\tau} \Omega(t, s) H(X(s ; x, t), s) d s .
\end{aligned}
$$

Invoking the $C^{1}$-smoothness, we have

$\lim _{\tau \rightarrow 0} \frac{T^{0}(X(t+\tau ; x, t), t+\tau)}{\tau}=\frac{\partial T^{0}}{\partial t}(x, t)+\frac{\partial T^{0}}{\partial x}(x, t) f(x, t)$

and

$$
\begin{aligned}
& \lim _{\tau \rightarrow 0} \frac{\Omega(t+\tau, t) T^{0}(x, t)}{\tau}=\left.\frac{d \Omega}{d t}(t, s)\right|_{s=t} T^{0}(x, t)=A(t) T^{0}(x, t) \\
& \lim _{\tau \rightarrow 0} \frac{1}{\tau} \Omega(t+\tau, t) \int_{t}^{t+\tau} \Omega(t, s) H(X(s ; x, t), s) d s=H(x, t) .
\end{aligned}
$$

Combining (34), we verify that $T^{0}(x, t)$ defined by (33) is a feasible solution to the PDE (32), and then

$$
\phi^{0}(x, t)=x+T^{0}(x, t)
$$

is a $C^{1}$-solution to $(25)$ in $\mathcal{X}$.

The next step is to verify the rank condition (28). We have

$$
\frac{\partial \phi^{0}}{\partial x}(x, t)=I_{n}+\frac{\partial T^{0}}{\partial x}(x, t)
$$

with

$$
H\left(x_{\star}, t\right)=-f\left(x_{\star}, t\right)+A x_{\star}=0, \quad \frac{\partial H}{\partial x}\left(x_{\star}, t\right)=0,
$$

and then $T\left(x_{\star}, t\right)=0$ and $\nabla T\left(x_{\star}, t\right)=0$. It implies the existence of a small parameter $\varepsilon>0$ such that the mapping $\phi^{0}(x, t)$ is an injection (fox any fixed $t$ ) in $B_{\varepsilon}\left(x_{\star}\right)$. Similarly to the proof of Theorem 2, we consider the flow in the $z$ coordinate as

$$
z\left(t_{x}\right)=\Omega\left(t_{x}, t\right) z(t)=\Omega\left(t_{x}, t\right) \phi^{0}(X(t ; x, s), t),
$$

as well as the flow in the $x$-coordinate

$$
z\left(t_{x}\right)=\phi^{0}\left(X\left(t_{x} ; x, s\right), t_{x}\right) .
$$

Hence, choosing $s=t$ and using $\left.X(t ; x, s)\right|_{s=t}=x$ and $\Omega\left(t_{x}, t\right) \Omega\left(t, t_{x}\right)=I$, we modify the transformation as

$$
\phi(x, t)=\Omega\left(t, t_{x}\right) \phi^{0}\left(X\left(t_{x} ; x, t\right), t_{x}\right),
$$

which satisfies both the PDE (25) and the immersion condition (28) with sufficiently large $t_{x}>0$.

In the above proof, we have assumed the existence of an equilibrium $x_{\star}$. Now, let us consider the case without equilibria. From the assumption of contraction, we know that all trajectories will converge to each other ultimately. Selecting a particular solution $x_{r}(t)$, we have

$$
\left|x(t)-x_{r}(t)\right| \leq k_{0}\left|x(0)-x_{r}(0)\right| e^{-\rho t}, \quad x(0) \in \mathbb{R}^{n}
$$


with some $k_{0}, \rho>0$. We define an error state $\chi:=x-x_{r}$, the dynamics of which is given by

$$
\dot{\chi}=f_{\chi}(\chi, t)
$$

with

$$
f_{\chi}(\chi, t)=f\left(\chi+x_{r}(t), t\right)-f\left(x_{r}(t), t\right) .
$$

Clearly, this system is GES at the origin due to (36). Then, we may apply the result with an equilibrium to the dynamics (37). The associated Koopman transformation is given by $\phi(x-$ $\left.x_{r}(t), t\right)$, where $\phi(\cdot, t)$ follows the construction above.

Sometimes there is an interesting special case to identify constant Koopman eigenvalues, or equivalently to lift the nonlinear model into an LTI system. It is always possible to do that, but imposing the immersion condition $\mathbf{C} 1$ requires that the Jacobian of the vector field should not change significantly over time. We have the following.

Proposition 3: Consider the NLTV system (2) with an equilibrium $x_{\star}$ at the origin, and assuming the system is contracting with a metric $M(x)$ in the set $c \mathcal{l}(\mathcal{X})$. There always exists a Koopman mapping $\phi(x, t)$ satisfying $\mathbf{C 1}^{\prime}$ but with a constant Hurwitz matrix $A \in \mathbb{R}^{n \times n}$. Further, if

$$
\left|\frac{\partial f}{\partial x}(0,0)-\frac{\partial f}{\partial x}(0, t)\right| \leq k, \quad \forall t \geq 0
$$

for some small $k>0$, then the condition $\mathbf{C 2}^{\prime}$ also holds.

Proof: From the contraction assumption, we have

$$
\dot{M}(x)+F^{\top}(x, t) M(x)+M(x) F(x, t) \leq-\gamma M(x)
$$

for some $\gamma>0$, with $F(x, t)=\frac{\partial f(x, t)}{\partial x}$. Since $x_{\star}=0$ is an equilibrium, we have $\dot{M}\left(X\left(x_{\star}, t\right)\right)=0$, thus

$$
F^{\top}\left(x_{\star}, t\right) M\left(x_{\star}\right)+M\left(x_{\star}\right) F\left(x_{\star}, t\right) \leq-\gamma M\left(x_{\star}\right) .
$$

It implies that the Jacobian $F\left(x_{\star}, t\right)$ is Hurwitz at any fixed time. Now we select the Hurwitz matrix $A:=F\left(x_{\star}, 0\right)$, which may work in our analysis due to the assumption (38). Now we consider $N=n$ and parameterize $\phi$ as

$$
\phi(x, t)=x+T(x, t)
$$

and define $H(x, t):=-f(x, t)+A x$.

The existence of a Koopman mapping $\phi$ is now equivalent to the existence of the solution to the PDE (25) which satisfies the immersion condition. With the above parameterization (39), the equation (25) becomes

$$
\frac{\partial T}{\partial t}(x, t)+\frac{\partial T}{\partial x}(x, t) f(x, t)=A T(x, t)+H(x, t) .
$$

It is interesting to figure out that the PDE (40) is exactly the same one in the KKL observer for non-autonomous systems $[3,4]$. Mimicking the operation done in (15), we may modify the NLTV system as $\dot{x}=\rho(x) f(x, t)$ with its solution denoted as $\breve{X}(s ; x, t)$. That is, $\breve{X}(s ; x, t)$ represents the solution value at time $s$ from the initial condition $x(t)$ at time $t$ for the modified dynamics. Then, the PDE (25) has a feasible solution

$$
T^{0}(x, t)=\int_{0}^{t} e^{A(t-s)} H(\breve{X}(s ; x, t), s) d s,
$$

which is well-posed from the backward complete property. Therefore, $\phi^{0}(x, t)=x+T^{0}(x, t)$ is a feasible solution to (25) in $\operatorname{cl}(\mathcal{X})$.

The remainder of the proof is to verify (28) under the additional assumption (38). The Jacobian of $\phi^{0}(x, t)$ is

$$
\frac{\partial \phi^{0}}{\partial x}(x, t)=I_{n}+\frac{\partial T^{0}}{\partial x}(x, t) .
$$

Noting that

$$
H\left(x_{\star}, t\right)=-f\left(x_{\star}, t\right)+A x_{\star}=0, \frac{\partial H}{\partial x}\left(x_{\star}, 0\right)=0
$$

it yields that $\phi^{0}(x, t)$ is an immersion in $B_{\varepsilon}\left(x_{\star}\right)$ for small $\varepsilon>0$, if $k>0$ is sufficiently small. Following the similar proof of Theorem 2, we redesign the mapping $\phi^{0}(x, t)$ as

$$
\phi(x, t)=e^{-A\left(t_{x}-t\right)} \phi^{0}\left(X\left(t_{x} ; x, t\right), t_{x}\right)
$$

by choosing a sufficiently large $t_{x}>0$. Hence, we have verified the immersion condition.

Remark 9: The additional assumption (38) is relatively mild. A particular case is the system dynamics being in the form of $\dot{x}=f_{1}(x)+f_{2}(t)$, which satisfies (38) automatically. In Proposition 3 and Theorem 4, we lift the contracting NLTV system (2) into an LTI and an LTV system, respectively. In the latter, we remove the Jacobian requirement (38) of the vector field $f(x, t)$. The key underlying reason relies on that in the latter we have $\frac{\partial H}{\partial x}\left(x_{\star}, t\right)=0, \forall t$, but for the former we only have $\frac{\partial H}{\partial x}\left(x_{\star}, 0\right)=0$ only at the initial moment without uniformity with respect to time.

\section{Extension to LIMIT CYCLES}

In this section, we show the equivalence between the transverse contraction and Koopman approaches in orbital stability analysis. Indeed, periodic behaviour plays important roles in many engineering and biological applications $[31,46,56]$. Given an autonomous system (1), a (non-trivial) periodic solution $X$ is one for which there exists $T>0$ such that

$$
X(t)=X(t+T), \quad t \geq 0,
$$

and the orbit is the set

$$
\gamma:=\left\{x \in \mathbb{R}^{n} \mid x=X(t), 0 \leq t \leq T\right\}
$$

Analogous to the results for equilibria, in [36, Proposition 3] the authors propose a Koopman operator-based stability criterion for limit cycles. Let us recall the results in [36], and we modify as follows. Without loss of generality, we assume the system invariant in the set $\mathcal{X}$.

Lemma 2: Consider the system (1) with a hyperbolic limit cycle $\gamma \subset \mathbb{R}^{n}$. If there exists a Koopman mapping $\phi(x):=$ $\left[\phi_{\lambda_{1}}(x), \ldots, \phi_{\lambda_{n-1}}(x)\right]^{\top} \in \mathcal{O}^{n-1}$ satisfying

T1 (immersion) $\Phi(x)=\frac{\partial \phi}{\partial x}(x)$ is full row rank uniformly in the set $\gamma \subset \mathcal{X} \in \mathbb{R}^{n}$, and $\left.\phi(x)\right|_{x \in \gamma}=0$;

T2 (stability) the existence of a Hurwitz matrix $A \in$ $\mathbb{R}^{(n-1) \times(n-1)}$ verifying the PDE (7).

Then, the system is orbitally asymptotically stable in $\mathcal{X}$ with respect to the limit cycle $\gamma$.

Due to the topological constraint, the set $\mathcal{X}$ cannot be the entire space of $\mathbb{R}^{n}$. The best result we may get in the Euclidean 
space is that the basin of attraction is almost global, except a zero Lebesgue measure set.

Meanwhile, [34, Thm. 3] provides a transverse contraction criterion for existence and stability of a limit cycle. See Appendix for the definition of transverse contraction.

Lemma 3: [34] Consider the system (1). If there exists a uniformly bounded metric $M(x) \in \mathbb{R}_{\succ 0}^{n \times n}$ such that

$$
\dot{M}+{\frac{\partial f(x)^{\top}}{\partial x}}^{\top} M+M \frac{\partial f(x)}{\partial x}-\rho(x) f(x) f(x)^{\top} \prec 0
$$

for some scalar function lower bounded $\rho(x)>0$, then the system is orbitally asymptotically stable.

The interested reader may refer to [34] for its convex representation. A benefit of Lemma 3, compared to Lemma 2 , is that it does not require the prior knowledge of the orbit $\gamma$ to verify orbital stability of a given nonlinear system. In [57], it was also suggested to use the bounded semi-definite Riemannian metric $M(x) \in \mathbb{R}_{\succeq 0}^{n \times n}$ to verify the transverse contraction with the inequality

$$
\dot{M}(x)+\mathbb{M}(x) \frac{\partial f(x)}{\partial x}+\frac{\partial f(x)^{\top}}{\partial x} \mathbb{M}(x) \prec-k M(x), \quad k>0 .
$$

Since $\mathbb{M}$ can be parameterized as $\mathbb{M}(x)=\Psi(x) P(x) \Psi^{\top}(x)$ with $P(x) \in \mathbb{R}_{\succ 0}^{r \times r}$ and $\Psi \in \mathbb{R}^{n \times r}$, if $\nabla \Psi_{i}=\left(\nabla \Psi_{i}\right)^{\top}$ for all $i=1, \ldots, r$, then the attractive orbit can be obtained as

$$
\gamma=\left\{x \in \mathbb{R}^{n} \mid \psi(x)=0\right\},
$$

with

$$
\psi(x):=\int_{0}^{1}(\Psi(s x))^{\top} x d s
$$

if $\left.\psi(x)\right|_{\gamma}=0$ with $r=n-1$.

\section{A. Koopman Implies Transverse Contraction}

We are now ready to show that the Koopman approach for limit cycles implies transverse contraction, i.e., verifying both (44) and the conditions in Lemma 3.

Theorem 5: Consider the system (1) satisfying the assumptions in Lemma 2. Then, the system is transversely asymptotically contracting with respect to $\phi$, i.e., there exist

(1) a metric $M(x) \succeq 0$ satisfying (44) globally;

(2) a metric $M(x) \succ 0$ satisfying (43) locally.

Proof: If there exists a Koopman mapping $\phi$ satisfying the conditions T1-T2, we can find a matrix $P \in \mathbb{R}_{\succ 0}^{r \times r}$ with $r:=n-1$ satisfying the Lyapunov inequality

$$
P A+A^{\top} P \preceq-k P, \quad k>0 .
$$

Then, we construct the semi-definite Riemannian metric as $M(x)=\frac{\partial \phi(x)}{\partial x}^{\top} P \frac{\partial \phi(x)}{\partial x}$. Following the similar procedure as in the proof of Theorem 1, we have

$$
\dot{M}+M F+F^{\top} M \preceq-k M,
$$

thus verifying the first sufficient condition in (44).

Then, we briefly summarize the construction of the transverse contraction metric to guarantee the conditions in Lemma 3 locally. Since $\Phi(x)=\frac{\partial \phi}{\partial x}(x)$ is full rank, invoking Wazewski theorem [3, Ch. 9.3], we know that the Jacobian completion of $\nabla \phi$ is solvable, i.e., there exists a $C^{\infty}$ mapping $\Theta: \mathbb{R}^{n} \rightarrow$ $\mathbb{R}^{1 \times n}$ such that

$$
\operatorname{det}(N(x)) \neq 0, \quad N(x):=\operatorname{col}(\Phi(x), \Theta(x))
$$

in any contractible sets. ${ }^{9}$ Then, we may select the positive definite metric $M(x)$ as

$M(x)=N(x)^{\top}\left[\begin{array}{ll}P & 0 \\ 0 & 1\end{array}\right] N(x)=\Phi(x)^{\top} P \Phi(x)+\Theta(x)^{\top} \Theta(x)$,

in which $P$ is the solution to the Lyapunov equation $P A+$ $A^{\top} P+I=0$. According to the results in [34, Thm. 4], it completes the proof.

\section{B. Transverse Contraction Implies the Koopman Condition}

Now let us show the converse result, i.e., transverse contraction implies the Koopman conditions for limit cycles. We make the following assumption.

Assumption 1: Consider a limit cycle $\gamma \in \mathcal{X} \subset \mathbb{R}^{n}$ with the transverse coordinate $\xi \in \mathbb{R}^{n-1}$ and the tangential coordinate $\theta \in \mathbb{S}$ defined by

$$
\left[\begin{array}{l}
\xi \\
\theta
\end{array}\right]=\phi_{1}(x):=\left[\begin{array}{l}
\phi_{\xi}(x) \\
\phi_{\theta}(x)
\end{array}\right]
$$

satisfying

$$
\left.\phi_{\xi}(x)\right|_{x \in \gamma}=0 .
$$

Additionally, $\nabla \phi_{1}(x)$ is full rank for $x \in \mathcal{X}$.

Theorem 6: Consider the system (1), which admits a (nontrivial) limit cycle $\gamma$ satisfying Assumption 1. If there exists a uniformly bounded matrix $P(x) \in \mathbb{R}_{\succ 0}^{(n-1) \times(n-1)}$ such that the semi-definite Riemannian metric $M(x)=$ $\nabla \phi_{\xi}(x) P(x)\left(\nabla \phi_{\xi}(x)\right)^{\top}$ satisfying (44), then there is a Koopman mapping $\phi: \mathbb{R}^{n} \rightarrow \mathbb{R}^{n-1}$ satisfying $\mathbf{T 1}$ and $\mathbf{T} 2$.

Proof: From the existence of a limit cycle, there are a family of particular solutions $x_{\star}(t)$ invariant on $\gamma$, i.e.

$$
x_{\star}(t)=x_{\star}(t+T) \in \gamma, \quad \forall t \geq 0
$$

for some $T>0$. According to [57, Proposition 11], we obtain

$$
\lim _{t \rightarrow \infty}\left\|\phi_{\xi}(X(x, t))\right\|_{\gamma}=0, \quad \forall x \in \mathcal{X}
$$

with $\|x\|_{\gamma}:=\inf _{y \in \gamma}|x-y|$ and the $\xi$-system dynamics

$$
\dot{\xi}=\left.\frac{\partial \phi_{\xi}}{\partial x} f(x)\right|_{x=\phi_{1}^{-1}(\xi, \theta)}
$$

is contracting in terms of the assumption (44) and has an equilibrium at the origin, where $\phi_{1}^{-1}$ is the inverse mapping of $\phi_{1}$. According to Theorem 4, there exists a transformation $\phi_{2}: \mathbb{R}^{n-1} \times \mathbb{R} \rightarrow \mathbb{R}^{n-1}$ lifting (46) into ${ }^{10}$

$$
\dot{\zeta}=A(\theta) \zeta,
$$

where $A$ is a stable (time-varying) matrix.

\footnotetext{
${ }^{9}$ If $\Theta(x)$ is additionally integrable, i.e. $\frac{\partial \theta}{\partial x}=\Theta$, then $\phi(x)$ and $\theta(x)$ represent the transverse and tangential coordinates (i.e. isochrons, or angular variable), respectively, for orbital stability analysis.

${ }^{10}$ The angular variable $\theta$ plays the role of time $t$ in Theorem 4 . Indeed, we may find a reversible function between $(\xi, \theta)$ and $(\xi, t)$; see for example the proof of [22, Thm 2.6].
} 
Note that $\theta$ is defined on $\mathbb{S}$ (via normalization), as well as $\left.\dot{\theta}\right|_{\gamma} \neq 0$, and thus $A(\theta)$ is a periodic, stable matrix. According to Floquet theorem, there is a periodic matrix $Q(\theta)$ such that the transformed coordinate $z=Q(\theta) \zeta$ has an LTI dynamics

$$
\dot{z}=A_{Q} z
$$

with $A_{Q} \in \mathbb{R}^{(n-1) \times(n-1)}$ Hurwitz.

As a result, the Koopman mapping is given by the composite function

$$
\phi(x):=Q\left(\phi_{\theta}(x)\right) \cdot \phi_{2} \circ \phi_{\xi}(x),
$$

completing the proof.

In the above result, we assume that we already know the transformation to get transverse and tangential coordinates. Indeed, the existence of such a transformation in its domain of attraction was shown in [7]. The converse result in Theorem 6 resembles the necessary part of [36, Proposition 3].

\section{DISCUSSIONS}

\section{A. Extension to Stabilization Problems}

We have showed the equivalence between the Koopman and contraction approaches when analyzing stability of equilibria, trajectories, and limit cycles. On the other hand, it is of practical interest to study if such equivalence holds in constructive problems.

Let us consider the controlled system model

$$
\dot{x}=f(x, u),
$$

with the control input $u \in \mathbb{R}^{m}$.

Definition 3: (control contraction metric) Consider the controlled system (48) with Jacobians $F(x, u):=\frac{\partial f(x, u)}{\partial x}$ and $G(x, u):=\frac{\partial f(x, u)}{\partial u}$. If we can find a uniformly bounded metric $M(x)$ and a function $K(x)$ satisfying

$$
\dot{M}+M F+F^{\top} M+M G K+(G K)^{\top} M \prec 0,
$$

then we call $M(x)$ a (strong) control contraction metric.

For affine-in-input systems, the CCM may be written in a more compact way independent of $K(x)$. The interested reader may refer to [32] for additional details.

Similar to the autonomous case, for the controlled system (48) we may define the Koopman mapping $\phi$ satisfying

$$
\frac{\partial \phi}{\partial x}(x) f(x, u)=A \phi(x)+B u, \forall u \in \mathbb{R}^{m},
$$

with matrix $B \in \mathbb{R}^{n \times m}$, which transforms the dynamics into

$$
\dot{z}=A z+B u, z(0)=\phi(x(0)) .
$$

We have the following.

Proposition 4: Consider the controlled system (48), which has a Koopman mapping $\phi$ satisfying $\mathbf{C 1}$ and the PDE (50). If the lifted LTI system (51) is stabilizable, then the given controlled system admits a CCM.

Proof: The stabilizability of the LTI system (51) is equivalent to the existence of a matrix $P=P^{\top} \succ 0$ and a feedback gain matrix $\bar{K}$ such that

$$
P(A+B \bar{K})+(A+B \bar{K})^{\top} P \prec 0 .
$$

Now we define the feedback controller $u=\bar{K} \phi(x)$, and substitute into the PDE (50), obtaining

$$
\Phi(x) f(x, \bar{K} \phi(x))=(A+B \bar{K}) \phi(x) .
$$

Calculate its partial derivative with respect to $x$, yielding

$$
\dot{\Phi}(x)+\Phi(x)[F+G \bar{K} \Phi(x)]=(A+B \bar{K}) \Phi .
$$

By selecting the metric

$$
M(x):=\Phi(x)^{\top} P \Phi(x)
$$

and the mapping $K(x):=\bar{K} \Phi(x)$, we have

$$
\begin{aligned}
& \Phi^{\top}\left[P(A+B \bar{K})+(A+B \bar{K})^{\top} P\right] \Phi \\
= & \dot{M}+M F+F^{\top} M+M G K+(G K)^{\top} M \\
\prec & 0,
\end{aligned}
$$

which exactly coincides with the strong CCM in (49).

Remark 10: Although Koopman and CCM methods for control design are equivalent in certain cases, they differ in their implementation and each have their advantages. In particular, CCM methods admit a convex search for the metric and the differential controller $K$, however there is no guarantee that the obtained $K(x)$ is integrable and some online computation may be required to realize a specific controller [24,53]. On the other hand, the joint search for observables $\phi$ and controller in the Koopman framework is non-convex, but if successful there always admits an explicit controller $u=K \phi(x)$.

Remark 11: In general, the lifted $z$-dynamics may contain a state-dependent input matrix rather than constant $B[12,15]$. Here, we use the constant input matrix assumption to simplify the presentation, as is popular in data-driven Koopman-based methods for control, e.g. [42], [37, Ch. 5]. A straightforward extension is the case with state-dependent $B(x)$, and in many cases we may parameterize $u=\alpha(x, v)$ and regard $v$ as the new input in order to get the form $\dot{z}=A z+B v$. On the other hand, noting that the input $u$ can be arbitrary, the PDE (50) imposes implicitly the affine-in-control assumption of $f(x, u)$, making it relatively restrictive. It is also promising to be extended to more general cases.

Remark 12: It is interesting to study the converse claim for stabilization problems. For an affine-in-control system $\dot{x}=$ $f(x)+g u$, if there exists a feedback law $u=k(x)+v(t)$ making the closed-loop system contracting, then following the main results of the paper, there is a mapping $\phi(x)$ which lifts the closed-loop into a stable linear system, and also lifts the open loop into a stabilizable dynamics. Unfortunately, it is not a bona fide converse, since a CCM controller may be not integrable to a function $k(x)$ with $K(x)=\frac{\partial k}{\partial x}(x)$.

\section{B. Relations to KKL Observers}

The KKL observer, also known as nonlinear Luenberger observer, is a state estimation approach for general nonlinear systems [16], which consists in mapping the given nonlinear dynamics

$$
\dot{x}=f(x, t), \quad y=h(x, t)
$$

to a linear system

$$
\dot{z}=A z+D y
$$


with $A$ Hurwitz, in which the output $y$ is viewed as an available signal. This step relies on solving the PDE

$$
\frac{\partial T}{\partial t}+\frac{\partial T}{\partial x}(x) f(x, t)=A T(x, t)+D h(x, t) .
$$

It is a quite general framework since it allows a nonlinear output injection $h(x, t)$ to appear in the transformed $z$-coordinate. The associated PDE (53) is always solvable under some mild technical assumptions, and when applying KKL observer, the main task is to guarantee the coordinate change $x \mapsto z=$ $T(x, t)$ injective, thus admitting an inverse mapping. It was shown that, the general notion - backward distinguishability is sufficient to guarantee the injectivity $[1,3]$. Some remarks about the relation between the Koopman method and KKL observers are in order.

1) In the proof of the converse results in Sections IV, we parameterize the coordinate change $\phi:(x, t) \mapsto z$ into two parts, i.e., $\phi(x, t)=x+T(x, t)$, and then we obtain (14) for autonomous systems and (40) for time-varying systems. It exactly coincides with the PDE involved in KKL observers for the auxiliary system

$$
\dot{x}=f(x, t), \quad y=H(x, t) .
$$

with $H(\cdot)$ the high-order remainder terms defined in Section IV. Using the constructive solution in KKL observers, we get a feasible solution to the PDE in the Koopman methods for nonlinear contracting systems.

2) The main difference, between the Koopman method and KKL observers, relies on how to guarantee the injectivity. For the latter, we need to show the injectivity of $T(x, t)$ by exploiting the backward distinguishability of the given system. However, for the Koopman method, we need to show the injectivity of $\phi(x, t)=x+T(x, t)$ rather than $T(x, t)$ itself. Our key idea is to utilize its "identity part".

3) In KKL observers, it is suggested to use excessive coordinates, generally more than $(2 n+1)$-dimensional ${ }^{11}$, in order to get the injectivity of the mapping $T$ defined in (41). On the other hand, excessive coordinates are widely adopted for the Koopman operator in the learning literature. It is claimed in [18, Thm. 3] that by choosing sufficiently rich orthonormal bases, the solution of a least square approximates the Koopman operator with guaranteed accuracy.

Remark 13: (Extensions to control design) An interesting open problem is the converse claim of Proposition 4, which is related to the dual problem of KKL observer. A straightforward idea is to immerse the controlled system (48) into

$$
\dot{z}=A z+h(x, u), \quad z=T(x)
$$

for some function $h(x, u)$ to be determined, with $A$ stable. In this step, we have the same PDE as the one in KKL observers. Then, the stabilization task generally contains two tasks:

- finding a function $\alpha(x)$ to solve the algebraic equation $h(x, \alpha(x))=0$;

- the function $h(x, t)$ should guarantee that the system $\dot{x}=$ $f(x, u), y=h(x, u)$ is backward distinguishable.

\footnotetext{
${ }^{11}$ For elements selected in $\mathbb{C}$, the dimension is not less than $n+1$.
}

Then, the feedback law $u=\alpha(x)$ stabilizes the system at some equilibrium.

\section{EXAMPLES}

\section{A. A 2-Dimensional System}

Consider the nonlinear autonomous system [6]

$$
\dot{x}=\left[\begin{array}{c}
-x_{1} \\
-x_{2}+x_{1}^{2}
\end{array}\right]
$$

with $x \in \mathbb{R}^{2}$. The differential dynamics of (55) is given by $\delta \dot{x}=F(x) \delta x$, with the Jacobian

$$
F(x)=\left[\begin{array}{cc}
-1 & 0 \\
2 x_{1} & -1
\end{array}\right] .
$$

By selecting the metric $M(x)=\operatorname{diag}\left(1+4 x_{1}^{2}, 1\right)$, we may verify that the given system is contracting due to

$\dot{M}(x)+M(x) F(x)+F(x)^{\top} M(x)=\left[\begin{array}{cc}-2-16 x_{1}^{2} & 2 x_{1} \\ 2 x_{1} & -2\end{array}\right] \prec 0$.

This example will be used to verify the converse result in Section III-B. The system has an equilibrium at the origin, i.e. $x_{\star}=0$, and then following the proof of Theorem 2 we have $F_{\star}=F\left(x_{\star}\right)=\operatorname{diag}(-1,-1)$. A feasible transformation is $\phi(x)=x+T(x)$, with $T(x)$ the solution of (17). The flow $X(x, t)$ of the given nonlinear system (55) can be obtained as

$$
X(x, t)=\left[\begin{array}{c}
e^{-t} x_{1} \\
e^{-t} x_{1}^{2}+e^{-t} x_{2}-e^{-2 t} x_{1}^{2}
\end{array}\right],
$$

and the high-order remainder term is $H(x)=\operatorname{col}\left(0,-x_{1}^{2}\right)$. We consider the modified system (15) in the the open set $\mathcal{X}:=$ $\left\{0<x_{1}<1\right\}$, and the backward flow of $\breve{X}_{1}$ is given by

$$
\breve{X}_{1}(x, t)=\left\{\begin{array}{lr}
e^{-t} x_{1}, & \ln x_{1} \leq t \leq 0 \\
1, & t<\ln x_{1} .
\end{array}\right.
$$

Then, it yields

$$
\begin{aligned}
T(x) & =\int_{0}^{+\infty} \exp \left(F_{\star} s\right) H(\breve{X}(x,-s)) d s \\
& =\int_{-\infty}^{0}\left[\begin{array}{ll}
e^{s} & \\
& e^{s}
\end{array}\right]\left[\begin{array}{c}
0 \\
-\breve{X}_{1}(x, s)^{2}
\end{array}\right] d s \\
& =-\int_{\ln x_{1}}^{0}\left[\begin{array}{c}
0 \\
e^{s}\left(e^{-s} x_{1}\right)^{2}
\end{array}\right] d s-\int_{-\infty}^{\ln x_{1}}\left[\begin{array}{c}
0 \\
e^{s} 1^{2}
\end{array}\right] d s \\
& =\left[\begin{array}{c}
0 \\
-2 x_{1}+x_{1}^{2}
\end{array}\right]
\end{aligned}
$$

thus

$$
\phi(x)=\left[\begin{array}{c}
x_{1} \\
-2 x_{1}+x_{1}^{2}+x_{2}
\end{array}\right] .
$$

It is straightforward to verify $\mathbf{C 1}$ and $\mathbf{C 2}$, i.e., $\frac{\partial \phi}{\partial x}(x)$ is full rank, and

$$
\stackrel{\cdot}{\phi(x)}=\left[\begin{array}{c}
-x_{1} \\
2 x_{1}-x_{1}^{2}-x_{2}
\end{array}\right]=F_{\star} \phi(x) .
$$

We underline here that these conditions hold globally in $\mathbb{R}^{2}$. It is interesting to compare the above result with the one in [6], in which the given system is immersed into a three-dimensional LTI system by introducing excessive coordinates. 


\section{B. Limit Cycle in Induction Motor}

In this section, we use the model of an induction motor to illustrate the result in Section V-A, i.e., the Koopman condition implies transverse contraction for limit cycles. The normalized model in the fixed frame is given by [41]

$$
\begin{aligned}
& \dot{\psi}_{r}=-R \phi_{r}+\omega \rrbracket \psi_{r}+R u \\
& \dot{\omega}=u^{\top} \rrbracket \psi_{r}-\tau_{L}, \quad \rrbracket:=\left[\begin{array}{cc}
0 & -1 \\
1 & 0
\end{array}\right],
\end{aligned}
$$

with the flux $\psi_{r} \in \mathbb{R}^{2}$, angular speed $\omega \in \mathbb{R}$, the load torque $\tau_{L} \in \mathbb{R}$, the resistance $R>0$, and the stator current $u \in \mathbb{R}^{2}$ as input. A basic control problem is to regulate the norm $\left|\psi_{r}\right|$ and the speed $\omega$ to some constants $\beta_{\star}$ and $\omega_{\star}$, respectively. To address this, the classical field-oriented control (FOC), which was introduced in the drives community in 1972 [5], is now the de facto standard in all high-performance applications of electric drives. With zero load $\tau_{L}$, the FOC takes the form

$$
u=\left[\beta_{\star} I_{2}-\frac{k}{\beta_{\star}}\left(\omega-\omega_{\star}\right) \rrbracket\right] \frac{\psi_{r}}{\left|\psi_{r}\right|}, \quad k>0 .
$$

We assume that - with loss of generality - all constant parameters and gains being one to simplify the presentation. In [56, Proposition 4] it shows that the FOC (57) achieves almost global orbital stabilization. The following gives an alternative proof from the aspect of Koopman operator.

Proposition 5: The induction motor model (56) in closed loop with (57) satisfies the assumptions in Lemma 2, and thus has an attractive limit cycle.

Proof: For consistency of notations, we define the state $x=\left[\psi_{r}^{\top}, \omega\right]^{\top} \in \mathbb{R}^{3}$ with $x_{p}=\left[x_{1}, x_{2}\right]^{\top}$. Then, the closed loop is given by

$$
\dot{x}=\left[\begin{array}{ccr}
1-\left|x_{p}\right| & -x_{3}\left|x_{p}\right| & \frac{x_{2}}{\left|x_{p}\right|} \\
* & 1-\left|x_{p}\right| & -\frac{x_{1}}{\left|x_{p}\right|} \\
* & * & -\left|x_{p}\right|
\end{array}\right] \nabla \mathcal{H}
$$

with “*” presenting some skew-symmetric elements, and the Hamiltonian $\mathcal{H}(x)=\frac{1}{2}\left|x_{p}\right|^{2}+\frac{1}{2}\left(x_{3}-1\right)^{2}$. Now, let use verify the assumptions for the closed loop (58). It is straightforward to verify that the Jordan curve $\gamma=\left\{x \in \mathbb{R}^{3}:\left|x_{p}\right|=1, x_{3}=\right.$ $1\}$ is forward invariant, and there is no equilibrium on the set. Let us select the Koopman eigenfunctions as

$$
\phi_{1}(x)=1-\frac{1}{\left|x_{p}\right|}, \quad \phi_{2}(x)=\frac{x_{3}-1}{\left|x_{p}\right|} .
$$

The Jacobian $\frac{\partial \phi}{\partial x}(x)$ is full rank almost globally, except the zero-Lebesgue measurable set $\Omega_{s}:=\left\{x \in \mathbb{R}^{3}: x_{1}=x_{2}=0\right\}$, verifying the condition T1. On the other hand, we have

$$
\overbrace{\phi(x)}^{\cdot}=-\phi(x)
$$

thus verifying T2, i.e., the PDE (7) with the Hurwitz matrix $A=-I_{2}$. Invoking Lemma 2, we complete the proof.

According to Theorem 5, there exists a transverse contraction metric $M(x)$ satisfying the inequality (43). To see this, we may select the matrix-valued function

$$
\Theta(x)=\left[\begin{array}{lll}
-x_{2} & x_{1} & 0
\end{array}\right]
$$

to guarantee the full-rank condition (45) almost globally except the singular set $\Omega_{s}$. By selecting $P=I_{2}$ we have $P A+A^{\top} P \prec 0$, and as a result the matrix

$$
M(x)=\left[\frac{\partial \phi}{\partial x}(x)\right] P\left[\frac{\partial \phi}{\partial x}(x)\right]^{\top}+\Theta(x)^{\top} \Theta(x)
$$

is positive definite except $\Omega_{s}$. In Fig. 3, we draw the largest

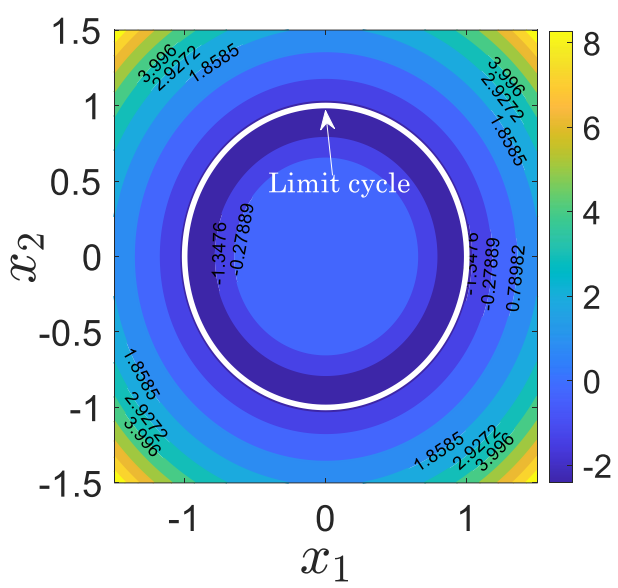

Fig. 3: The largest real parts of the eigenvalues of $\dot{M}+$ $\frac{\partial f}{\partial x}^{\top} M+M \frac{\partial f}{\partial x}-\rho f f^{\top}$ via fixing $x_{3}=1$

real parts of the eigenvalues of $\dot{M}+\frac{\partial f}{\partial x}^{\top} M+M \frac{\partial f}{\partial x}-\rho f f^{\top}$ with $\rho=50$, and note that for the purpose of visualization we fix $x_{3}$ at its steady-state value $x_{3}=1$. Clearly, $M(x)$ verifies the transverse contraction condition (43) in the neighborhood of the limit cycle .

Remark 14: In the above analysis, we use explicitly the equation of the limit cycle $\gamma$ to illustrate the theoretical results in Section V-A. However, it is unnecessary to write down the analytic form of $\gamma$ via transverse contraction to find a limit cycle and prove its stability [34]. This is particularly important for showing robustness of a limit cycle, since typically a limit cycle location will change as parameters change.

\section{Learning Contraction Metrics from Data}

It is widely recognized that the Koopman operator provides a powerful tool to learn dynamical models of nonlinear systems from data $[18,28,30,37]$. Based on the equivalence between contraction and Koopman approaches studied in the paper, it can provide a novel approach to learn contraction metrics for stable nonlinear systems from pure trajectory data. Learning contraction metrics has recently been explored in the context of robust motion planning and control, e.g. [8, 48, 51], and our results dramatically simplify this problem to one of linear system identification.

Problem. (Data-driven contraction metrics learning) Consider a contracting system (1) and assume that only a set of state trajectory data $\{\tilde{x}(k)\}_{k=0}^{T}$ and the derivatives $\{\dot{\tilde{x}}(k)\}_{k=0}^{T}$ are available ${ }^{12}$, and the vector field $f(x)$ is unknown. Our task is to estimate the contraction metric $M(x)$ using the information of data only.

\footnotetext{
${ }^{12}$ Sometimes the time derivative of systems state is not available from sensors. We may apply stable filters to obtain new regressors.
} 
According to Theorem 2, if the system is contracting we may always find a Koopman mapping $\phi$ to get a lifted LTI system (8) with $A$ Hurwitz, and $\phi$ is left invertible. A contraction metric of the given system is $M=\nabla \phi P \nabla \phi^{\top}$ with $P \succ 0$ the solution of the Lyapunov equation. Based on this intuitive idea, data-driven contraction metrics learning is translated to the problem of estimating the Koopman mapping $\phi$ and the stable matrix $A$ from data.

First, selecting the basis function $w(x) \in \mathbb{R}^{N}$, with $N \in \mathbb{N}$, and here $N>n$ is usually selected sufficiently large in order to get high accuracy. Then, we are able to parameterize the Koopman mapping as

$$
\phi(x)=\theta^{\top} w(x), \quad \theta \in \mathbb{R}^{N \times n} .
$$

Now the PDE (7) becomes

$$
\theta^{\top} \overparen{w_{(x)}}=A \theta^{\top} w(x)
$$

with $\dot{w}(x)=W(x) \dot{x}$ and $W(x):=\frac{\partial w}{\partial x}(x)$. Our target, then, becomes searching for $\theta$ and $A$ in the optimization problem

$$
\begin{array}{ll}
\min _{\theta, A} & \sum_{k=1}^{n_{k}}\left|\theta^{\top} Y_{1}(k)-A \theta^{\top} Y_{2}(k)\right|^{2} \\
\text { s.t. } & \operatorname{rank} \theta=n
\end{array}
$$

with the measurable vectors $Y_{1}(k):=W(\tilde{x}(k)) \dot{\tilde{x}}(k)$ and $Y_{2}(k):=w(\tilde{x}(k))$.

We consider the following assumption. If we select sufficient numbers of independent basis functions $(N \gg n)$, all the elements of $W(x) f(x)$ can be approximately linearly represented by the bases $w(x)$, i.e. $[W(x) f(x)]_{i}=\rho_{i}^{\top} w(x)+$ $O(\cdot), \forall i$ for some vectors $\rho_{i}$, with some (tiny) high-order term $O(\cdot)$. Since $\theta$ is full rank, we always can find another matrix $\theta_{\perp} \in \mathbb{R}^{N \times(N-n)}$ such that $\left[\theta, \theta_{\perp}\right]$ is full rank. Hence,

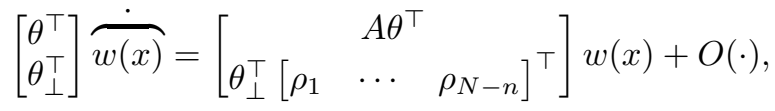

and then we have

$\left.\stackrel{\cdot}{\widetilde{w(x)}}=\tilde{A} w(x)+O(\cdot), \tilde{A}:=\left[\begin{array}{l}\theta^{\top} \\ \theta_{\perp}^{\top}\end{array}\right]^{-1}\left[\begin{array}{lll}\theta_{\perp} & A \theta^{\top} \\ \theta_{\perp}^{\top} & \cdots & \rho_{N-n}\end{array}\right]^{\top}\right]$.

Now we may estimate the matrix $\tilde{A}$ using the bases $w(x)$ as observables to identify the dynamical model. This is the underlying reason why, in many works, only (sufficiently highdimensional) lifted system matrix $\tilde{A}$ are learned - rather than estimating the Koopman mapping $\phi$ and matrix $A$ simultaneously - but it still provides satisfactory identification results. Then we may use the least square solution to estimate $\tilde{A}$ as $\hat{A}=\tilde{Y}_{1} \tilde{Y}_{2}^{\top}\left(\tilde{Y}_{2} \tilde{Y}_{2}^{\top}\right)^{-1}$ with $\tilde{Y}_{1}:=\left[\begin{array}{lll}Y_{1}(1) & \cdots & Y_{2}(k)\end{array}\right]$ and $\tilde{Y}_{2}:=\left[\begin{array}{lll}Y_{2}(1) & \cdots & Y_{2}(k)\end{array}\right]$. After solving $\hat{Q} \hat{A}+\hat{A}^{\top} \hat{Q}=$ $-I_{N}$, we learn a contraction metric as $M=\frac{\partial w}{\partial x} \hat{Q}^{\top} \frac{\partial w}{\partial x}$.

Numerical example. Let us consider the system in Section VII-A. But, now we only have the dataset $\{\tilde{x}(k), \dot{\tilde{x}}(k)\}_{k=0}^{T}$ rather than the model itself. The basis function is selected as polynomials $w(x)=\operatorname{col}\left(x_{1}, x_{2}, x_{1} x_{2}, x_{1}^{2}, x_{2}^{2}\right)$. The trajectory data are collected under the sampling time $0.2 \mathrm{~s}$ from the initial condition $\left[\begin{array}{ll}-2 & 1\end{array}\right]^{\top}$. Following the above, the estimated contraction metric $M(x) \in \mathbb{R}^{2 \times 2}$ is obtained as

$M(x)=W(x)^{\top}\left[\begin{array}{ccccc}0.45 & 0.23 & 0.03 & -0.04 & 0.04 \\ * & 0.44 & -0.01 & -0.13 & -0.04 \\ * & * & 0.23 & 0.1 & 0.12 \\ * & * & * & 0.29 & 0.07 \\ * & * & * & * & 0.23\end{array}\right] W(x)$.

We give in Fig. 4 the largest real part of the eigenvalues of $\dot{M}+M F+F^{\top} M$, which is negative definite - showing that the Riemmanian metric is geodesically decreasing - and the smallest real part of the eigenvalue of $M(x)$, which is positive definite. Hence, the obtained estimation of $M(x)$ is qualified as a contraction metric. It should be kept in mind that, for a given contracting system, the contraction metric is not unique.

Remark 15: We use this example to show an interesting byproduct of the main results in the paper, i.e., the Koopman approach provides an efficient methodology to learn contraction metrics from trajectory data. Some remarks are in order.

- By using sufficiently high-dimensional basis observables, we try to learn the matrix $\tilde{A}$ in the $w$-coordinate, which admits a modelling error from the high-order approximation term. If we come back to (61) and search for $A$ and $\phi$, it is promising to improve accuracy. It, however, is a non-convex optimization problem.

- The above procedure is sensitive to the quantity and coverage of data, with the obtained contraction metric local to the trajectory data. A possible approach is to impose stability constraint of the matrix $A$ during identification, e.g. via the methods of $[21,29,52]$ towards robust learning algorithms. Recently, we have extended these equivalence results from continuous-time to discrete-time models in [10], leading to a nonlinear system identification approach with the model stability constraint. We refer the reader to see more practical examples therein.

\section{Concluding Remarks}

In this paper, we study the connections among the Koopman method, contraction analysis and KKL observers. First, we show that the conditions in the Koopman method implies contraction of a given autonomous or time-varying system by substitution of differential equations. Later, the converse results for both equilibria and limit cycles are proven under mild technical assumptions, the success of which relies on the interesting observation that the Koopman method enjoys the same PDE as the one in KKL observers. We also studied the nonlinear controlled systems, showing that stabilizability of the obtained linear systems implies the existence of a CCM of the original nonlinear system. In terms of the above results, it is reasonable to expect a further integration of these methods as a systematic constructive tool in the near future.

\section{APPENDIX}

\section{A. Definition of Transverse Contraction}

The concept of transverse contraction was proposed in [34] for analysis of limit cycles. In [57], it was generalized to deal with observer design. 


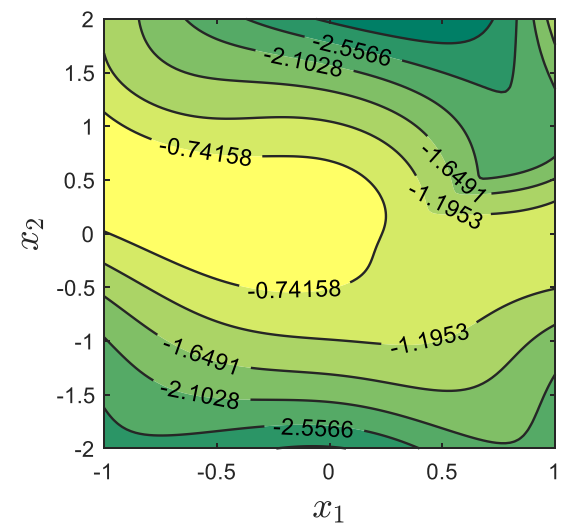

(a) The largest (real parts of) eigenvalue of $\left(\partial_{f} M+M F+F^{\top} M\right)$

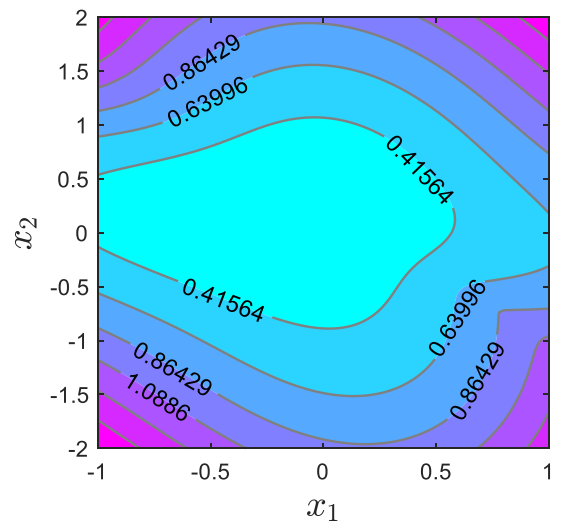

(b) The smallest (real parts of) eigenvalue of $M$

Fig. 4: Learned contraction metric from trajectory data

Definition 4: The forward complete system (1) is said to be transversely contracting with rate $\lambda>0$ (or transversely asymptotically contracting) with respect to $\psi: \mathbb{R}^{n} \rightarrow \mathbb{R}^{n}(1 \leq$ $r \leq n)$, if for any pair $\left(x_{a}, x_{b}\right) \in \mathbb{R}^{n} \times \mathbb{R}^{n}$, we have

$$
\left|\psi\left(X\left(x_{a}, t\right)\right)-\psi\left(X\left(x_{b}, t\right)\right)\right| \leq e^{-\lambda t} b\left(x_{a}, x_{b}\right)
$$

form some function $b \geq 0$ with $b(x, x)=0$ or

$$
\left|\psi\left(X\left(x_{a}, t\right)\right)-\psi\left(X\left(x_{b}, t\right)\right)\right| \leq \kappa\left(\left|x_{a}-x_{b}\right|, t\right)
$$

for transverse asymptotic contraction, with $\kappa$ of class $\mathcal{K} \mathcal{L}$.

The key difference between transverse contraction and horizontal contraction [11] is that the they are defined on state-space and tangent space, respectively. When adopting transverse contraction to the stability analysis of limit cycles [34], it becomes the existence of a Finsler-Lyapunov function $V(x, \delta x)$ satisfying

$$
\frac{\partial V(x, \delta x)}{\partial x} f(x)+\frac{\partial V(x, \delta x)}{\partial \delta x} \frac{\partial f(x)}{\partial x} \delta x \preceq-\lambda V(x, \delta x),
$$

for all non-zero $\delta x$ such that $\frac{\partial V}{\partial \delta x} f(x)=0$.

\section{ACKNOWLEDGMENTS}

The authors would like to express their gratitude to three reviewers for their thoughtful comments that helped improve its clarity, as well as Reviewer 5 of the conference version [55] for clarification of Remark 8 and Proposition 1.

\section{REFERENCES}

[1] V. Andrieu and L. Praly. On the existence of a KazantzisKravaris/Luenberger observer. SIAM J. Control Optim., 45(2):432-456, 2006.

[2] D. Angeli. A Lyapunov approach to incremental stability properties. IEEE Trans. Autom. Control, 47(3):410-421, 2002.

[3] P. Bernard. Observer Design for Nonlinear Systems. Lecture Notes in Control and Information Sciences. Springer, 2019.

[4] P. Bernard and V. Andrieu. Luenberger observers for nonautonomous nonlinear systems. IEEE Trans. Autom. Control, 64(1):270-281, 2019.

[5] F. Blaschke. The principle of field orientation as applied to the new transvektor closed-loop control system for rotating field machines. Siemens Rev., 34(1), 1972.

[6] S. L. Brunton, B. W. Brunton, J. L. Proctor, and J. N. Kutz. Koopman invariant subspacs and finite linear representations of nonlinear dynamical systems for control. PLOS ONE, 11(2):e0150171, 2016.
[7] C. I. Byrnes. Topological methods for nonlinear oscillations. Notices of the AMS, 57(9):1080-1091, 2010.

[8] G. Chou, N. Ozay, and D. Berenson. Model error propagation via learned contraction metrics for safe feedback motion planning of unknown systems. ArXiv Preprint, 2021. arXiv:2104.08695.

[9] R. F. Curtain and H. Zwart. An Introduction to Infinite-Dimensional Linear Systems Theory, volume 21. Springer, 2012.

[10] F. Fan, B. Yi, D. Rye, G. Shi, and I. R. Manchester. Learning stable Koopman embeddings. In Proc. Amer. Control Conf., 2022. (See also arXiv:2110.06509).

[11] F. Forni and R. Sepulchre. A differential Lyapunov framework for contraction analysis. IEEE Trans. Autom. Control, 59(3):614-628, 2014.

[12] D. Goswami and D. A. Paley. Bilinearization, reachability, and optimal control of control-affine nonlinear systems: A Koopman spectral approach. IEEE Trans. Autom. Control, 2021.

[13] P. Hartman. A lemma in the theory of structural stability of differential equations. Proc. Am. Math. Soc., 11(4):610-620, 1960.

[14] W. P. M. H. Heemels, M. K. Camlibel, and M. F. Heertjes. Oblique projected dynamical systems and incremental stability under state constraints. IEEE Control Syst. Lett., 4(4):1060-1065, 2020.

[15] B. Huang, X. Ma, and U. Vaidya. Feedback stabilization using Koopman operator. In Proc. IEEE Conf. Decis. Control, pages 6434-6439, 2018.

[16] N. Kazantzis and C. Kravaris. Nonlinear observer design using Lyapunov's auxiliary theorem. Syst. Control. Lett., 34(5):241-247, 1998.

[17] B. O. Koopman. Hamiltonian systems and transformation in Hilbert space. PNAS, 17(5):315-318, 1931.

[18] M. Korda and I. Mezic. Linear predictors for nonlinear dynamical systems: Koopman operator meets model predictive control. Automatica, 93:149-160, 2018.

[19] M. Korda and I. Mezić. Optimal construction of Koopman eigenfunctions for prediction and control. IEEE Trans. Autom. Control, 65(12):5114-5129, 2020.

[20] M. D. Kvalheim and S. Revzen. Existence and uniqueness of global Koopman eigenfunctions for stable fixed points and periodic orbits. Phys. D: Nonlinear Phenom., page 132959, 2021.

[21] S. L. Lacy and D. S. Bernstein. Subspace identification with guaranteed stability using constrained optimization. IEEE Trans. Autom. Control, 48(7):1259-1263, 2003.

[22] Y. Lan and I. Mezic. Linearization in the large of nonlinear systems and Koopman operator spectrum. Phys. D: Nonlinear Phenom., 242(1):4253, 2013.

[23] A. Lasota and M. C. Mackey. Chaos, Fractals, and Noise: Stochastic Aspects of Dynamics, volume 97. Springer, 1994.

[24] K. Leung and I. R. Manchester. Nonlinear stabilization via control contraction metrics: A pseudospectral approach for computing geodesics. In Proc. Am. Control Conf., pages 1284-1289, 2017.

[25] D. C. Lewis. Metric properties of differential equations. Amer. J. Math., 71(2):294-312, 1949.

[26] T. Lin and H. Zha. Riemannian manifold learning. IEEE Trans. Pattern Anal. Mach. Intell., 30(5):796-809, 2008.

[27] W. Lohmiller and J.-J. E. Slotine. On contraction analysis for non-linear systems. Automatica, 34(6):683-696, 1998. 
[28] S. Macesic, N. Crnjaric-Zic, and I. Mezic. Koopman operator family spectrum for nonautonomous systems. SIAM J. Appl. Dyn. Syst., 17(4):2478-2515, 2018.

[29] J. M. Maciejowski. Guaranteed stability with subspace methods. Syst. Control. Lett., 26(2):153-156, Sept. 1995.

[30] G. Mamakoukas, I. Abraham, and T. D. Murphey. Learning data-driven stable Koopman operators. ArXiv Preprint, 2020. arXiv: 2005.04291.

[31] I. R. Manchester, U. Mettin, F. Iida, and R. Tedrake. Stable dynamic walking over uneven terrain. Int. J. Robot. Res., 30(3):265-279, 2011.

[32] I. R. Manchester and J. E. Slotine. Control contraction metrics: Convex and intrinsic criteria for nonlinear feedback design. IEEE Trans. Autom. Control, 62(6):3046-3053, 2017.

[33] I. R. Manchester and J. E. Slotine. Robust control contraction metrics: A convex approach to nonlinear state-feedback $\mathcal{H}_{\infty}$ control. IEEE Control Syst. Lett., 2(3):333-338, 2018.

[34] I. R. Manchester and J.-J. E. Slotine. Transverse contraction criteria for existence, stability, and robustness of a limit cycle. Syst. Control. Lett. 63:32-38, 2014

[35] A. Mauroy and I. Mezic. A spectral operator-theoretic framework for global stability. In 52nd Proc. IEEE Conf. Decis. Control, pages 5234 5239, 2013.

[36] A. Mauroy and I. Mezic. Global stability analysis using the eigenfunctions of the Koopman operator. IEEE Trans. Autom. Control, 61(11):3356-3369, 2016.

[37] A. Mauroy, Y. Susuki, and I. Mezić. The Koopman Operator in Systems and Control. Springer, 2020.

[38] I. Mezic. Spectral properties of dynamical systems, model reduction and decompositions. Nonlinear Dyn, 41(1):309-325, 2005.

[39] I. Mezic. Koopman operator spectrum and data analysis. ArXiv Preprint, 2017. arXiv:1702.07597.

[40] I. Mezic and A. Banaszuk. Comparison of systems with complex behavior. Phys. D: Nonlinear Phenom., 197(1):101-133, 2004.

[41] R. Ortega, J. A. L. Perez, P. J. Nicklasson, and H. J. Sira-Ramirez. Passivity-Based Control of Euler-Lagrange Systems: Mechanical, Electrical and Electromechanical Applications. Springer, 2013.

[42] J. L. Proctor, S. L. Brunton, and J. N. Kutz. Generalizing Koopman theory to allow for inputs and control. SIAM J. Appl. Dyn. Syst. 17(1):909-930, 2018.

[43] W. J. Rugh. Linear System Theory. Prentice-Hall, Inc., Upper Saddle River, New Jersey, 2 edition, 1996.

[44] S. Sastry. Nonlinear Systems: Analysis, Stability, and Control, volume 10. Springer, 2013.

[45] P. J. Schmid. Dynamic mode decomposition of numerical and experimental data. J. of Fluid Mechanics, 656:5-28, 2010.

[46] A. Shiriaev, J. Perram, and C. Canudas-de Wit. Constructive too for orbital stabilization of underactuated nonlinear systems: Virtual constraints approach. IEEE Trans. Autom. Control, 50(8):1164-1176, 2005.

[47] S. Singh, S. M. Richards, V. Sindhwani, J.-J. E. Slotine, and M. Pavone Learning stabilizable nonlinear dynamics with contraction-based regularization. Int. J. Robot. Res., 40(10-11):1123-1150, 2021.

[48] D. Sun, S. Jha, and C. Fan. Learning certified control using contraction metric. In Proc. Conf. Robot Learning, pages 1519-1539, 2020.

[49] M. M. Tobenkin, I. R. Manchester, and A. Megretski. Convex parameterizations and fidelity bounds for nonlinear identification and reducedorder modelling. IEEE Trans. Autom. Control, 62(7):3679-3686, 2017.

[50] M. Tomás-Rodríguez and S. P. Banks. Linear, Time-varying Approximations to Nonlinear Dynamical Systems: with Applications in Control and Optimization, volume 400. Springer, 2010.

[51] H. Tsukamoto, S.-J. Chung, and J.-J. E. Slotine. Neural stochastic contraction metrics for learning-based control and estimation. IEEE Control Syst. Lett., 5(5):1825-1830, 2020.

[52] J. Umenberger, J. Wågberg, I. R. Manchester, and T. B. Schön. Maximum likelihood identification of stable linear dynamical systems. Automatica, 96:280-292, 2018.

[53] R. Wang and I. R. Manchester. Continuous-time dynamic realization for nonlinear stabilization via control contraction metrics. In Proc. Amer. Control Conf., pages 1619-1624, 2020.

[54] X. Wang. A global Hartman-Grobman theorem. ArXiv Preprint, 2020. arXiv: 2002.06094

[55] B. Yi and I. R. Manchester. On the equivalence of contraction and Koopman approaches for nonlinear stability and control. In 60th Proc. IEEE Conf. Decis. Control, pages 4609-4614, 2021.

[56] B. Yi, R. Ortega, D. Wu, and W. Zhang. Orbital stabilization of nonlinear systems via Mexican sombrero energy shaping and pumpingand-damping injection. Automatica, 112:108661, 2020.
[57] B. Yi, R. Wang, and I. R. Manchester. Reduced-order nonlinear observers via contraction analysis and convex optimization. IEEE Trans. Autom. Control, 2021. 